\title{
Integrated bioinformatics analysis reveals dynamic candidate genes and signaling pathways involved in the progression and prognosis of diffuse large B-cell lymphoma
}

\author{
Alice Charwudzi ${ }^{\text {Equal first author, } 1}$, Ye Meng ${ }^{\text {Equal first author, } 1}{ }^{1}$, Linhui Hu ${ }^{1}$, Chen Ding ${ }^{1}$, Lianfang Pu ${ }^{1}$, Qian Li $^{1}$, Mengling Xu $^{1}$, \\ Zhimin Zhai ${ }^{\text {Corresp., } 1}$, Shudao Xiong ${ }^{\text {Corresp. } 1}$ \\ ${ }^{1}$ Department of Hematology/Hematological Lab, The Second Hospital of Anhui Medical University, Hefei, Anhui, China \\ Corresponding Authors: Zhimin Zhai, Shudao Xiong \\ Email address: zzzm889@163.com, xshdao@ahmu.edu.cn
}

Background. Diffuse large B-cell lymphoma (DLBCL) is a highly heterogeneous malignancy with varied outcomes. However, the fundamental mechanisms remain to be fully defined. Aim. We aimed to identify core differentially co-expressed hub genes and perturbed pathways relevant to the pathogenesis and prognosis of DLBCL. Methods. We retrieved the raw gene expression profile and clinical information of GSE12453 from the Gene Expression Omnibus (GEO) database. We used integrated bioinformatics analysis to identify differentially co-expressed genes. The CIBERSORT analysis was also applied to predict tumor-infiltrating immune cells (TIICS) in the GSE12453 dataset. We performed survival and ssGSEA (single-sample Gene Set Enrichment Analysis) (for TIICs) analyses and validated the hub genes using GEPIA 2 and an independent GSE31312 dataset. Results. We identified 46 differentially co-expressed hub module genes in the GSE12453 dataset. Gene expression levels and survival analysis found 15 differentially co-expressed core hub genes. The core genes prognostic values and expression levels were further validated in the GEPIA 2 database and GSE31312 dataset to be reliable $(p<0.01)$. The core genes' main KEGG (Kyoto encyclopedia of genes and genomes) pathway enrichments were Ribosome and Coronavirus disease - COVID-19. High expressions of the 15 core hub genes had prognostic value in DLBCL. The core genes showed significant predictive accuracy in distinguishing DLBCL cases from non-tumor controls, with the area under the curve (AUC) ranging from 0.992 to 1.00 . Finally, CIBERSORT analysis on GSE12453 revealed immune cells, including activated memory CD4+ T cells and M0, M1, and M2- macrophages as the infiltrates in the DLBCL microenvironment. Conclusion. Our study found differentially coexpressed core hub genes and relevant pathways involved in ribosome and COVID-19 disease that may be potential targets for prognosis and novel therapeutic intervention in DLBCL. 
1 Integrated bioinformatics analysis reveals dynamic candidate genes and signaling

2 pathways involved in the progression and prognosis of Diffuse Large B-Cell Lymphoma.

3

4 Alice Charwudzi ${ }^{1 \#}$, Ye Meng ${ }^{1 \#}$, Linhui $\mathrm{Hu}^{1}$, Chen Ding ${ }^{1}$, Lianfang $\mathrm{Pu}^{1}$, Qian $\mathrm{Li}^{1}$, Mengling Xu ${ }^{1}$

5 Zhimin Zhai $^{1}$, Shudao Xiong ${ }^{1}$

6

$7 \quad$ \# AC and YM should be considered joint first authors; the authors contributed equally to this

8 work.

9

${ }^{1}$ Department of Hematology/Hematological Lab, The Second Hospital of Anhui Medical

University, Hefei 230601, Anhui Province, People's Republic of China

Corresponding Authors:

Prof Shudao Xiong ${ }^{1}$

678 Furong Rd, Hefei, Anhui Province, 230601, People's Republic of China

Email address: xshdao@ahmu.edu.cn or

Prof Zhimin Zhai ${ }^{1}$

678 Furong Rd, Hefei, Anhui Province, 230601, People's Republic of China

Email address:Email: zzzm889@163.com 
23 Abstract

24 Background. Diffuse large B-cell lymphoma (DLBCL) is a highly heterogeneous malignancy

25 with varied outcomes. However, the fundamental mechanisms remain to be fully defined.

26 Aim. We aimed to identify core differentially co-expressed hub genes and perturbed pathways

27 relevant to the pathogenesis and prognosis of DLBCL.

Methods. We retrieved the raw gene expression profile and clinical information of GSE12453 from the Gene Expression Omnibus (GEO) database. We used integrated bioinformatics analysis to identify differentially co-expressed genes. The CIBERSORT analysis was also applied to predict tumor-infiltrating immune cells (TIICs) in the GSE12453 dataset. We performed survival and ssGSEA (single-sample Gene Set Enrichment Analysis) (for TIICs) analyses and validated the hub genes using GEPIA 2 and an independent GSE31312 dataset.

Results. We identified 46 differentially co-expressed hub module genes in the GSE12453 dataset. Gene expression levels and survival analysis found 15 differentially co-expressed core hub genes. The core genes prognostic values and expression levels were further validated in the GEPIA 2 database and GSE31312 dataset to be reliable $(\mathrm{p}<0.01)$. The core genes' main KEGG (Kyoto encyclopedia of genes and genomes) pathway enrichments were Ribosome and Coronavirus disease - COVID-19. High expressions of the 15 core hub genes had prognostic value in DLBCL. The core genes showed significant predictive accuracy in distinguishing DLBCL cases from non-tumor controls, with the area under the curve (AUC) ranging from 0.992

42 to 1.00. Finally, CIBERSORT analysis on GSE12453 revealed immune cells, including activated 
43 memory $\mathrm{CD}^{+} \mathrm{T}$ cells and $\mathrm{M} 0, \mathrm{M} 1$, and $\mathrm{M} 2$ - macrophages as the infiltrates in the DLBCL

44 microenvironment.

45 Conclusion. Our study found differentially co-expressed core hub genes and relevant pathways

46 involved in ribosome and COVID-19 disease that may be potential targets for prognosis and

47 novel therapeutic intervention in DLBCL. 


\section{Introduction}

Diffuse large B-cell lymphoma (DLBCL) is exceptionally heterogeneous and the most common aggressive non-Hodgkin lymphoma (NHL) subtype in adults. It is increasingly appreciated that its varied outcomes depend on the patients' clinical and biological features (Karube et al., 2018; W. Liu et al., 2019; Luo, Gu, Wang, Chen, \& Peng, 2018; Naresh et al., 2011). Despite several reports on the mechanism of DLBCL, its pathogenesis characterized by multiple abnormalities at different molecular levels remains unresolved. Its development and progression are multifaceted, comprising various signaling pathways and driver genes. Despite improved clinical outcomes with current therapies, such as rituximab-chemotherapy (R-CHOP), chimeric antigen receptor (CAR)-T cell, and advancement in stem cell transplantation, over $40 \%$ of high-risk patients relapse or develop the primary refractory disease. Mortality figures remain high (Karube et al., 2018; Luo et al., 2018). Therefore, an in-depth understanding of disease biology could reveal novel biomarkers of diagnostic and prognostic value. It will also facilitate the design of alternative personalized therapeutic strategies for DLBCL.

Advances in gene profiling technologies, high-throughput data, and bioinformatics databases make screening DLBCL for differentially co-expressed genes indispensable, particularly when integrated with personalized genomic profile data (Lui et al., 2015; van Dam, Võsa, van der Graaf, Franke, \& de Magalhães, 2018). Recently, Liu et al. identified eleven genes associated with endometrial cancer progression and prognosis by comprehensive bioinformatics analysis (J. Liu et al., 2019). Zhou et used CIBERSORT and other bioinformatics analyses for colon cancer. They found that the tumor microenvironment (TME) was abundantly enriched with M0 and M2 macrophages, activated memory CD4 ${ }^{+} \mathrm{T}$ cells, and other immune cells that could 
88 play crucial roles as biomarkers (R. Zhou et al., 2019). However, few integrated bioinformatics

89 studies have compared the gene expression profile of DLBCL with non-cancer controls.

90 Thus, we downloaded the Gene Expression Omnibus (GEO) raw dataset of GSE12453 and

91 compared 11 DLBCL cases with 24 non-cancer controls (non-neoplastic B lymphocytes isolated

92 from blood or tonsils). We performed a series of screens and analyses, including filtering off

93 differentially expressed genes (DEGs), enrichment analysis, and co-expression analysis to

94 determine hub genes of clinical significance to DLBCL. We identified 15 differentially co-

95 expressed core hub genes associated with the prognosis of DLBCL (RPS24, RPS21, RPL31,

96 RPL30, RPS17, MRPS28, FAU, RPS25, RPL22L1, NDUFA6, CXCL9, CCL4, MRPL33,

97 HEBP1, and RPL11). The KEGG (Kyoto encyclopedia of genes and genomes) analysis

98 associated most of the genes with Ribosome and Coronavirus disease - COVID-19 pathways.

99 Validation in the GEPIA 2 database and GSE31312 dataset revealed that the core genes had

100 consistent expression levels and were reliable. Receiver operating characteristic (ROC) curves

101 plotted demonstrated that the core genes could be potential diagnostic biomarkers. The identified

102 genes could play critical roles in diagnosis, prognosis and help establish a foundation for

103 developing or identifying novel targeted therapies for DLBCL.

104

105 Materials and methods

106 Data collection

107 We evaluated and downloaded the raw gene expression profiles from the National Center for

108 Biotechnology Information - Gene Expression Omnibus (NCBI-GEO) database

109 (https://www.ncbi.nlm.nih.gov/geo/). The series was based on GPL570 [HG-U133_Plus_2]

110 Affymetrix Human Genome U133 Plus 2.0 Array. GSE12453 (Brune et al., 2008) was used to 
111 identify differentially expressed and co-expressed genes. It was also used to predict tumor-

112 infiltrating immune cells (TIICs). It contained 11 DLBCL cases and 25 normal controls. The

113 controls were non-neoplastic B lymphocytes isolated from healthy donors' blood or tonsils from

114 routine tonsillectomy patients. Similar expression profiles on the selected GPL570 platform

115 contained normal but reactive controls or non-human controls, such as cell lines or limited

116 controls, and were excluded. We used the GSE31312 (Visco et al., 2012) with 498 DLBCL cases

117 for survival analysis and identifying immune cells infiltrating the TME.

\section{Study design and data pre-processing}

120 The GSE12453 CEL file was pre-processed using the Affymetrix package (Gautier, Cope,

121 Bolstad, \& Irizarry, 2004) in R software (https://www.r-project.org/) version 4.0.2 (Team, 2019).

122 The procedures included background correction, $\log _{2}$ transformation, followed by quantile

123 normalization. We performed a standard quality assessment, including scaling factors and NUSE

124 plots, and hierarchical clustering to identify outliers. The study's design flowchart is shown in

125 Fig S1.

\section{Screening of differentially expressed genes (DEGs)}

128 The DEGs between DLBCL cases and non-cancer controls were screened with R package limma

129 (https://www.bioconductor.org/), Release 3.11 (Ritchie et al., 2015). The cutoff criteria were

$130 \mathrm{p}<0.05, \mid \log _{2}$ fold change (FC) $\mid>1.0$. The pheatmap package was used to generate hierarchical

131 clustering and ggplot2 (Wickham, 2016) to show volcano plot in R. We used the resulting data

132 output tables that included gene ID, $\log _{2} \mathrm{FC}$, unadjusted and adjusted p-values in subsequent

133 analyses. 


\section{Functional and pathway enrichment analysis}

135 We investigated the functional roles and pathway signaling relevance of the DEGs. The gene

136

137

138

139

140

141

142

143

144

145

146

147

148

149

150

151

152

153

154

155

156

ontology (GO) and Kyoto encyclopedia of genes and genomes (KEGG) pathway enrichment analysis was performed using the R clusterprofiler (https://bioconductor.org/), Release 3.12 (Yu, Wang, Han, \& He, 2012). A p-value $<0.05$ was considered significantly enriched. The GO categories included biological process (BP), molecular function (MF), and cellular component (CC).

\section{Gene set enrichment analysis (GSEA)}

We used the normalized expression dataset of GSE12453 for the GSEA (www.gseamsigdb.org/gsea/index.jsp), version 4.1.0. We followed the recommended protocol. This included "gct" and "cls" file formats for the expression dataset and phenotype labels, respectively (Subramanian et al., 2005). Significant gene sets had false discovery rate (FDR) $<0.25$ and a nominal p-value $<0.05$.

\section{Protein-protein interaction (PPI) network of DEGs and hub modules selection}

We applied the STRING database (https://string-db.org), version 11.0 (Szklarczyk et al., 2019), to assess and map the identified DEGs into a human PPI network. Then, we uploaded the resulting data output into Cytoscape software (https://cytoscape.org/), version 3.8.2 (Shannon et al., 2003), and used MCODE with default parameters (Bader \& Hogue, 2003) and the Cytohubba (Chin et al., 2014) plug-ins to select significant modules and top-ranked genes. Nodes and edges represented genes and their interactions. 
157 Predicting tumor-infiltrating immune cells (TIICs)

158 We applied the Cibersort algorithm (Newman et al., 2019) in R to predict TIICs using the

159

160

161

162

163

164

165

166

167

168

169

170

171

172

173

174

175

176

177

178

179 normalized GSE12453 dataset, according to the CIBERSORT instructions. We used data with a p-value $<0.05$ for further analysis.

\section{Co-expression network construction and identification of modules related to DLBCL}

We conducted gene co-expression analysis on the processed GSE12453 data using the weighted correlation network analysis (WGCNA) (Peter Langfelder \& Horvath, 2008; P. Langfelder \& Horvath, 2012) in R (Team, 2019). We followed the standard protocols, including quality control procedures. We performed a power $\beta$ transformation on the computed Pearson correlation matrix to ensure a scale-free topology. The minimum number of module genes was set at 30 . The WGCNA R package then generated a co-expression network from the resulting adjacency matrix. We applied the dynamic Tree Cut package (Team, 2019) to create the co-expression modules from the color-coded hierarchical clustering dendrogram. We assessed clinical moduletrait relationships with Pearson's correlation. Gene significance (GS) and module membership (MM) were also analyzed for their correlation in modules. Statistically significant modules were defined as $\mathrm{p}<0.05$.

\section{Identifying differentially co-expressed hub module genes}

The hub genes were identified from the intersection between the DEGs and genes significant in the WGCNA modules. We then analyzed the hub genes with the STRING and Cytoscape databases. We further conducted functional and pathway analysis on the hub genes to determine the relevant genes that impact DLBCL. 
180

181

182

183

184

185

186

187

188

189

190

191

192

193

194

195

196

197

198

199

200

201

202

\section{Validation and survival analysis of core hub genes}

We validated the expression levels of the hub genes in the GEPIA2 (gepia2.cancer-

pku.cn/\#index) database (Tang, Kang, Li, Chen, \& Zhang, 2019). We retrieved the pre-processed quantile normalized series matrix file (GSE31312, Affymetrix HG-U133 Plus 2.0 GeneChips) containing 498 de-novo adults DLBCL from the NCBI-GEO database. We used it for the prognostic value analysis (overall survival (OS) and progression-free survival (PFS)). We plotted Kaplan-Meier survival curves with ggplot 2 in $\mathrm{R}$. The genes with p-value $<0.05$ were selected as core hub genes. Also, receiver operating characteristic (ROC) curves were plotted with the pROC R package (Team, 2019) to validate the diagnostic value of the core genes. Then, the human protein atlas (proteinatlas.org/) was used to confirm their protein expressions in some selected lymph node samples (Uhlen et al., 2017).

We used the ssGSEA (single-sample Gene Set Enrichment Analysis) package (Subramanian et al., 2005) in R on the GSE31312 dataset to investigate immune-associated core hub genes in the TME.

In addition, we also employed the GEPIA 2 and Oncomine (Rhodes et al., 2004) databases to determine the expression levels and relevance of the core hub genes in other tumors.

\section{Results}

\section{Differentially expressed genes (DEGs)}

According to standard protocols, one poor quality control sample (GSM312887) was excluded from the GSE12453 dataset after pre-processing. We identified 1260 DEGs between the 11 
203 DLBCL and 24 non-tumor controls. They comprised 1014 up-regulated and 246 down-regulated

204 genes $\left(\mathrm{p}<0.05\right.$ and $\left.\left|\log _{2} \mathrm{FC}\right|>1\right)$. The gene list, Affymetrix probe ID, and log FC are shown in

205 (Excel S1). The expression patterns of the DEGs are shown in Fig 1. Figure 1A shows the

206 volcano plot of all expressed genes. The DLBCL cases showed a distinctive gene expression

207 profiling. As shown in Fig. 1B, the heatmap of the top 169 DEGs with $\left|\log _{2} \mathrm{FC}\right|>2$ suggested

208 that the identified DEGs expression levels could differentiate DLBCL from non-tumor samples.

209 We utilized the CytoHubba application in Cytoscape, employing five calculation methods: the

210 Maximal Clique Centrality (MCC), Maximum Neighborhood Component (MNC), Degree, Edge

211 Percolation Component (EPC), and EcCentricity to rank the top 250 DEGs. The genes from the 5

212 methods were intersected using the Venn diagram software

213 (http://bioinformatics.psb.ugent.be/webtools/Venn/). Most of the intersecting genes (Fig. 1C)

214 were associated with significant and valuable pathways such as the proteasome, spliceosome,

215 and viral protein interaction with cytokine and cytokine receptor (Fig. S2). Intersecting genes are

216 common genes with a high degree of interconnection and are more likely to represent key

217 candidate genes with important biological regulatory functions.

218

219 Functional and pathway analysis

220 We applied the enrichGO or enrichKEGG function of the clusterProfiler package (Yu et al., 221 2012) in $R$ to investigate the biological functions of all the DEGs $(p<0.05)$. The GO and KEGG

222 pathway analysis results are shown in Fig. 2. In GO analysis, the DEGs were primarily enriched

223 in ATP synthesis coupled electron transport and mitochondria ATP synthesis coupled electron

224 transport for biological processes (BP). Their cellular components (CC) were mainly related to

225 the mitochondria protein complex, mitochondria inner membrane, etc. Their molecular functions 
226 (MF) consisted of structural constituents of ribosomes and NADH dehydrogenase activity (Fig.

227 2A). The KEGG pathway analysis showed significant enrichment in ribosome and oxidative

228 phosphorylation (Fig. 2B). Strikingly, the DEGs involved in the KEGG pathway and GO

229 enrichment analysis were mainly upregulated genes, with few down-regulated genes.

230 Further analysis showed that the down-regulated genes were enriched in B cell activation (adjust

231 p-value $=0.003$ ) for GO-BP; our data showed no other significant GO and KEGG enrichment for

232 the down-regulated genes. The GO and KEGG enrichment analysis for the upregulated genes

233 was similar to the analysis we performed earlier. The GO and KEGG analysis classifies genes

234 into functional categories to help understand their functions and regulatory pathways. Hence,

235 with additional investigations, the genes in these pathways might throw more light on the

236 pathogenesis of DLBCL.

237

Gene Set Enrichment Analysis (GSEA) of DLBCL expression dataset (GSE12453)

239

240

241

242

243

244

245

246

247
We performed a GSEA analysis to compare the DLBCL and non-tumor controls' expression profiles to understand better the biological functions of the relevant genes discovered. We analyzed all the qualified genes in the GSE12453 expression dataset. The KEGG output from the GSEA was similar to our previous pathway analysis and confirmed our earlier results. The Hallmark gene sets showed immune and metabolic-related signaling predominance, including estrogen response late, epithelial-mesenchymal transition, and UV response up (Table S1). Interestingly, genes defining late response to estrogen, epithelial-mesenchymal transition (such as in fibrosis and metastasis), and genes upregulated in response to ultraviolet (UV) radiation have not been fully elucidated in DLBCL. However, evidence suggests these genes have 
248 essential roles in oncogenesis. These findings provide evidence that can drive future research

249 with therapeutic implications.

250

251 Predicting tumor-infiltrating immune cells (TIICs)

252 Our GO, KEGG, and GSEA analyses showed that the DEGs were enriched in some immune-

253 related biological functions. Immune cell infiltration into tumors plays an essential role in

254 tumorigenesis and metastasis. So, we applied the CIBERSORT algorithm to the GSE12453

255 dataset to predict immune cells infiltrating the TME. Among the immune subsets analyzed,

256 activated memory $\mathrm{CD}^{+} \mathrm{T}$ cells, $\mathrm{CD}^{+} \mathrm{T}$ cells, and $\mathrm{M} 0, \mathrm{M} 1$, and $\mathrm{M} 2$ macrophages were the most

257 represented cell fractions within the DLBCL microenvironment (Fig. 3 A, B). The correlations

258 among the TIICs ranged from high to negligible (Fig. S3). Regulatory T cells (Tregs) showed a

259 moderate negative correlation with activated memory $\mathrm{CD}^{+} \mathrm{T}$ cells. However, M0 macrophages

260 had a high positive correlation with activated memory $\mathrm{CD}^{+} \mathrm{T}$ cells and $\mathrm{M} 1$ macrophages. The

261 varied infiltrating immune cell types could be reflective of the complexity and the unusual

262 behavior of DLBCL. Uncommitted macrophages (M0) can polarize into the M1 (considered

263 tumoricidal) and M2 (pro-tumorigenic) to show paradox effects on tumor prognosis (Dancsok et

264 al., 2020). Memory B cells and activated dendritic cells were the most represented fractions in

265 the non-tumor controls (Fig. 3 A). The findings suggest that TIICs may be closely associated

266 with clinical outcomes. Future studies, including their correlation to DLBCL disease stages, will

267 be meaningful, particularly for immunotherapy.

268

269 Protein-protein interaction (PPI) network and hub modules establishment

270 We constructed the PPI network of all DEGs using the STRING database's multiple proteins

271 function to determine genes likely to perform biological functions together. The highest 
272 confidence of 0.9 was set with unconnected nodes taken out. As shown in Fig. 4A, it yielded 609

273 nodes and 8583 edges. These genes were highly inter-connected than expected (PPI enrichment

274 p-value $<1.0 \mathrm{e}-16)$. We observed 6 important clusters with a k-score $>10$ when we analyzed the

275 tab-separated values (tsv) file with Cytoscape's MCODE. The largest cluster (\#1) had the highest

276 score of 33.17 (Fig. 4B); it mainly comprised Ribosome genes (FDR=3.49e-83) in KEGG

277 analysis. Cluster 2 (Fig. 4C) was enriched in genes associated with oxidative phosphorylation

278 (FDR, 7.23e-56) and Parkinson's disease (FDR, 2.62e-55). Cluster 3 (Fig. 4D) genes were

279 mainly involved in chemokine signaling (FDR, 1.06e-22). We identified 109 highly connected

280 DEGs (Excel S1) from these top 3 clusters. These DEGs could play essential roles in DLBCL, so

281 they were selected for further hub gene screening.

282

283 In this study, the down-regulated DEGs were not part of the constructed PPI, so we determined

284 their relevance in DLBCL. We ranked the top 60 DEGs (30 up- and 30 down-regulated) by

$285 \log _{2}$ FC and analyzed them in the STRING database (Fig. S4A). The only down-regulated DEG in

286 the network built was identified upregulated when verified in the GEPIA2 database. To further

287 investigate the down-regulated DEGs' functional relatedness, a PPI was constructed for all the

288 down-regulated DEGs (Fig. S4B). We found that the down-regulated genes were enriched in B

289 cell activation for GO-BP, shown in (Fig. S4C). Finally, we used the degree method of the

290 CytoHubba application to predict the top 250 important genes (Fig. 4E). Genes with a high

291 degree of centrality are vital since they have many direct interacting gene partners. If confirmed,

292 these critical findings could improve the general understanding and the potential causes of

293 variation in the clinical prognosis of DLBCL. 


\section{Weighted gene co-expression network (WGCNA) analysis}

296 To identify co-expression modules that could share similar biological functions or regulatory

297 mechanisms with clinical relevance to DLBCL, we applied the WGCNA package (Peter

298 Langfelder \& Horvath, 2008; P. Langfelder \& Horvath, 2012) in R (Team, 2019). The

299 GSE12453 dataset we processed was used. We carried out quality control procedures, including

300 inspecting good genes and sample hierarchical clustering to detect potential outliers but no

301 obvious outliers (Fig. 5 A). The 35 samples yielded two main clusters. We applied the WGCNA

302 on the top $25 \%$ of the 21654 expressed genes ranked by the largest variance. To satisfy a scale-

303 free network topology, we choose the soft-threshold power $\beta$ of 8 with $\mathrm{R} 2=0.86$ (Fig. 5 B, C).

304 Hierarchical clustering and the dynamic tree-cutting yielded 18 modules of co-expressed genes

305 (Fig. 5D). Finally, we visualized the top 1000 significantly expressed genes with a heatmap (Fig.

$3065 E)$; they represent interesting genes for further analysis.

307

308 To investigate the molecular mechanisms of the traits, we correlated each Module Eigengenes

309 (ME) to disease status (DLBCL and non-tumor controls). The results are shown in Fig. 6. The

310 ME turquoise and green (Fig. 6 A, B) containing 292 and 72 genes, respectively, strongly

311 correlated with DLBCL. ME dark magenta with 5 genes had the strongest negative correlation.

312 The cut-off was set at gene significance (GS) value $>0.8$, and absolute Module Membership

$313(\mathrm{MM})$ value $\geq 0.7$. Besides, the GS versus MM plots for these 3 modules were highly correlated

314 (Fig. 6C), reflecting their high association with DLBCL. We selected these 3 clinically

315 significant modules with the 369 high connectivity genes (gene list shown in Excel S1) for

316 further analysis. The highly connected genes are often the most important (central) elements of

317 the respective modules and tend to play key roles in the biological processes. The ME genes are 
318 listed in Excel S1. Altogether, these co-expressed genes might provide new clues to understand

319 the biology of DLBCL in the future.

320

321 PPI and functional enrichment analysis of the WGCNA relevant modules

322 The 369 high connectivity genes from the 3 relevant modules were filtered in the STRING

323 database followed by the Cytoscape; the network yielded a PPI with 195 nodes and 1579 edges.

324 The PPI and gene list are detailed in Fig. S5 and Excel S1. These 195 genes were considered

325 functionally important. As presented in (Fig. 7), functional annotation revealed that these genes

326 were involved in viral transcription and viral gene expression in the BP category. In KEGG

327 analysis, the genes were primarily enriched in ribosome, coronavirus disease - COVID-19, and

328 oxidative phosphorylation. The identified pathways were roughly consistent with that of the

329 DEGs. These processes and signaling pathways are usually disrupted in cancer and could provide 330 an insight into the pathogenesis of DLBCL.

331

\section{Identification of hub genes and pathways}

333 Eventually, we identified 46 important differentially co-expressed genes by the Venn diagram 334 software, as shown in (Fig. 8A). These 46 genes were common between the DEGs and WGCNA 335 hub module genes and were regarded as hub genes. We re-analyzed the 46 genes with the 336 STRING and Cytoscape databases, and the PPI is shown in (Fig. 8B). Their GO and KEGG 337 enrichments in the R software (Table 1) were similar to the other analyses. The KEGG common 338 genes in the ribosome, COVID-19, and oxidative phosphorylation pathways are shown in Table

2. The above pathway genes play essential roles in metabolic reprogramming and tumor-

340 promoting inflammation of cancer and warrant further studies. 
342 Validation of expressions and prognostic analysis of core hub genes

343 We applied GEPIA 2 to validate the reliability and authenticity of the 46 hub genes in the cancer

344 genome atlas (TCGA) dataset. We identified 44 prognostic genes with higher expression

345 (consistent with that in the GSE12453 dataset) in DLBCL tissues than the non-tumor control

346 tissues $(\mathrm{p}<0.01)($ Fig. 9) $(15$ genes shown) and (Excel S1). Kaplan-Meier survival analysis on the

347 GSE31312 showed 15 of these genes $(\mathrm{p}<0.05)$ correlated with patient outcomes (Fig. 10 \& Table

348 S2). Except for RPL11, the patients with high expressions had significantly shorter 5-year OS

349 and PFS, suggesting these genes are potential oncogenes and have a role in DLBCL development

350 and/or progression. The 15 genes (Fig. 11A) were considered as the core hub genes. Moreover,

351 ROC curve analysis for their diagnostic potentials obtained AUCs ranging from 0.992 to 1.00,

352 indicating optimal performance to accurately differentiate DLBCL from non-tumor control cases

353 (Fig. S6). Also, immunohistochemistry data from the human protein atlas (HPA) database

354 demonstrated the protein expressions of some of the genes in some lymph node samples with

355 cytoplasmic/ membranous localization (Fig. S7, 4 genes shown); data were retrieved from

356 https:/www.proteinatlas). The genes included RPS21, MRPS28, RPL31, and RPL30. As

357 expected, they would be involved in metabolic pathways such as glycolysis and processes

358 including signal transduction and cell division.

359 The core hub genes' functional annotation was mainly associated with Ribosome and

360 Coronavirus disease - COVID-19 (Fig. 11 B, C; Table 3). To assess the tumorigenic potentials of

361 the COVID-19 genes regarding immune cells infiltrating the TME, the ssGSEA analysis was

362 applied (GSE31312). As shown in Fig. S8, 7 out of the 9 COVID-19 pathway genes negatively

363 correlated with mast cells, 5 with immature dendritic cell (iDC), and 3 genes negatively 
364 correlated with plasmacytoid DC. RPL30, RPL31, RPS25, and FAU positively correlated with 365 tumor-infiltrating lymphocytes (TIL) and macrophages. RPL30, RPL31 correlated with Tregs.

366 These infiltrating immune cells may be involved in regulating tumor proliferation, dormancy, 367 and drug resistance.

368

369 Finally, we determined whether the core genes were upregulated in other tumors. The GEPIA 2 370 database revealed that all the 15 core genes were upregulated in thymoma (THYM), and 11

371 genes were upregulated in testicular germ cell tumors (TGCT). Notably, RPL30 and FAU genes

372 were consistently upregulated in all 6 different cancer types identified (Table S3). In the

373 Oncomine database, some of the core genes were upregulated in various lymphoma datasets and 374 other cancers, including Sarcoma (Fig. S9). The results suggest that the upregulation of these 15 375 hub genes may not be limited to DLBCL.

376

\section{Discussion}

378 Diffuse large B-cell lymphoma (DLBCL) remains a significant clinical challenge; over $30 \%$ of 379 patients are not cured (Pasqualucci \& Dalla-Favera, 2018; Yi et al., 2020). So far, no functional assays capable of screening exit, so effective management is required once diagnosed. Hence, identifying unique gene signatures and regulatory pathways related to its pathogenesis and prognosis is meaningful. Here, we examined the gene expression profile of GSE12453 to find dysregulated common core hub genes and pathways to help further understand DLBCL pathogenesis and provide potential biomarkers. differentially co-expressed core hub genes linked to DLBCL pathogenesis. The genes included 
RPS24, RPS21, RPL31, RPL30, RPS17, MRPS28, FAU, RPS25, RPL22L1, NDUFA6, CXCL9,

389 CCL4, MRPL33, HEBP1, and RPL11. Their primary KEGG enrichment was ribosome and

390

391

392

393

394

395

396

397

398

399

400

401

402

403

404

405

406

407

408

409

410

coronavirus disease - COVID-19, which was in line with the other analyses. The construction of

ROC curves yielded very high AUC values suggesting the genes could accurately distinguish

between DLBCL and non-tumor control cases and might be potential biomarkers. In addition, experimentally derived data from the HPA by IHC indicated the protein expression of some of the genes in some lymph node samples. RPS21, MRPS28, RPL31, and RPL30 showed relatively higher protein expressions in some DLBCL and other malignant lymphoma tissues than the averaged expressions in normal tissues, though not significant. The HPA database sample size was limited; however, the HPA experimental findings can be extended to DLBCL and other lymphomas, thus providing a valuable basis for medical and biological research.

Lastly, most of the core genes were upregulated in different cancer types. Cancer is a complex disease, so the genes might have similar or different prognostic roles in these tumors. The overall survival data from GEPIA 2 demonstrated that low levels of FAU, RPS17, and RPS 24 were significantly associated with shorter survival, while high CCL4 was significantly associated with shorter survival in thymoma patients. Nonetheless, the genes' potential biological and clinical relevance is not restricted to only DLBCL. These genes could be prognostic markers and therapeutic targets across different tumor types, particularly for patients with multiple coexisting tumors.

Little is known experimentally about the roles of most of the core genes proteins in DLBCL. However, dysregulated ribosomal proteins have been reported to play various critical roles in 
411 other tumors (Wang et al., 2015). Among our ribosome genes, over-expressed RPS21 promoted

412 prostate cancer (PCa) cell proliferation, migration, and invasion, inhibited PCa cell apoptosis,

413 and was suggested as a promising biomarker, with a potential application in diagnosis or

414 treatment (Liang et al., 2019). The 8q-mapped RPL30 gene was associated with adverse

415 outcomes in Medulloblastoma patients (De Bortoli et al., 2006). RPS24 significantly promoted

416 colorectal cancer (CRC) cells' proliferation rate and increased CRC risk in patients (Zou et al.,

417 2020). The knockdown of RPS24 inhibited cell proliferation and cell migration in human CRC

418 cell lines and was recommended as a biomarker (Wang et al., 2015). A study also implicated

419 MRPS28 in the molecular pathogenesis of bladder cancer (Liu et al., 2021).

420

421 The coronavirus disease - COVID-19 and viral transcription enrichments agree with recent

422 studies implicating various viruses (Gandhi et al., 2020) (Fedoriw et al., 2020) in the

423 development and progression of DLBCL subtypes. Most DLBCL patients have an underlying

424 immune dysfunction and can easily get viral infections. Viruses such as COVID 19 could

425 manipulate the function of the COVID 19 related genes in the TME. Besides, the Gene Cards

426 database (https://www.genecards.org/) demonstrated all the covid 19 pathway genes (RPS24,

427 RPS21, RPL31, RPL30, RPS17, FAU, RPS25, RPL22L1, RPL11) are related to viral mRNA

428 translation (Stelzer et al., 2016). Additionally, the DAVID database (Protein interactions)

429 associated 5 COVID 19 genes (RPL30, RPS17, RPS 24, RPS 25, and FAU) with HIV

430 interactions (Huang da, Sherman, \& Lempicki, 2009).

431

432 Recently, hematological malignancies (HM) patients were reported to have a more severe

433 COVID-19 trajectory than patients with solid organ tumors (Lee et al., 2020). A significant 
434 number of the COVID 19 related genes were upregulated in various lymphomas and some

435 multiple myeloma datasets (Fig. S9). Most of our COVID-19 pathway genes showed some

436 correlations with immune infiltrates such as TIL and macrophages. An anti-viral immune

437 response can have protective effects with improved survival in coronavirus infection, but

438 excessive inflammation can be harmful ("cytokine storm"). High pro-inflammatory macrophage

439 (M1) and low $\mathrm{CD}^{+} \mathrm{T}$ cells were observed in the microenvironment of severe/critical COVID-19

440 patients (Liao et al., 2020). Higher expression of CXCL9 in COVID-19 patients than healthy

441 controls and higher levels of CCL4 in severe COVID-19 patients were also found (Liao et al.,

442 2020). These are partly consistent with our data and previous knowledge on various cancers

443 (Brune et al., 2008; Chang et al., 2013; De la Fuente López et al., 2018). Not much is known

444 about COVID-19 and most cancers, including DLBCL pathogenesis. However, it is tempting to

445 speculate that COVID-19 infection, together with the COVID-19 related genes, could increase

446 macrophage polarization to M1 (hyper-inflammatory response) to worsen prognosis (He et al.,

447 2020; Passamonti et al., 2020; Shah et al., 2020). But, the mechanism is unclear, and limited data

448 on the topic did not permit detailed discussion. However, extensive genome-related studies are

449 required to verify this association between COVID 19 and DLBCL; these genes could provide a

450 basis to identify effective preventive and therapeutic strategies.

451

452 Clinical implication analysis in the GEPIA2 database showed that the core hub genes were

453 significantly overexpressed in DLBCL. The high expressions of 14 (93\%) were negatively

454 associated with prognostic outcomes (worse OS and PFS times). These emphasize their potential

455 role as oncogenes and could be utilized as prognostic indicators for DLBCL. The high

456 expression of RPL11 might be associated with a favorable clinical outcome (Kawahata et al.,

457 2020; Kayama et al., 2017). They offer unique opportunities for further investigation. 
458 In addition to the ribosome (translation), the 46 hub genes were significantly represented in

459 oxidative phosphorylation (OxPhos) and mitochondria inner membrane. Growing evidence

460 suggests cancer is primarily a mitochondrial metabolic disease that exhibits altered energy

461 production and dysregulated metabolic crosstalk (Yin et al., 2019) (Norberg et al., 2017). Their

462 inhibition has demonstrated anti-cancer efficacy (Martínez-Reyes et al., 2020) (Norberg et al.,

463 2017). Thus, with further studies, these metabolic genes could be rational targets, especially for

464 the metabolically coupled and OxPhos-DLBCL subsets, and help understand the metabolic

465 differences in DLBCL.

466

467 The few bioinformatics analyses on DLBCL focused on the subtypes (Huang, Liu, \& Shen, 468 2019; L. Zhou et al., 2020) or clinical features (Xiao, Wang, \& Bai, 2020). However, some 469 dysregulated genes specific to DLBCL versus non-tumor controls cannot distinguish the 470 subtypes (Huang et al., 2019). Moreover, most related studies that focused on DLBCL and non471 cancer controls were based on DEGs (Huang et al., 2019; Luo et al., 2018) and discovered 472 entirely different core hub genes. However, the complexity of DLBCL and the emergence of 473 novel targeted therapies warrants more predictive personalized biomarkers for precision 474 medicine. To our knowledge, no integrated bioinformatics analysis on DLBCL and non-tumor 475 controls has so far been reported on the common core hub genes found and immune cells 476 associations. Thus, our finding is novel.

477 One potential limitation of this study is the lack of experimental validation. However, this 478 analysis provides a theoretical basis for our future work, which will focus on experimental 479 verification. Second, an individual study with limited DLBCL cases was used, but the hub genes, 
480 pathways, and immune cells infiltrate identified are relevant to the pathogenesis of DLBCL and

481 cannot be ignored. However, our results should be interpreted with caution.

482

483

\section{Conclusions}

484

485

486

487

488

489

490

491

492

493

494

495

496

497

498

499

500

501

502

503

504

505

506

507

508

509

510

511

512

We used the integrated bioinformatics method to highlight the critical roles of differentially coexpressed core hub genes and relevant pathways in DLBCL. We identified some immune-related core hub genes linked to DLBCL pathogenesis. The core genes' main KEGG pathway

enrichments were Ribosome and Coronavirus disease - COVID-19. Their verification in GEPIA 2 showed they were reliable. Nevertheless, most of the core genes were upregulated in different cancer types and hold potential biological and clinical relevance in cancers. Thus, the identified genes could be potential targets for prognosis and therapeutic intervention in DLBCL and may provide insight into the pathogenetic mechanisms in DLBCL.

\section{References}

Bader, G. D., \& Hogue, C. W. (2003). An automated method for finding molecular complexes in large protein interaction networks. BMC Bioinformatics, 4, 2. doi:10.1186/1471-2105-4-2

Brune, V., Tiacci, E., Pfeil, I., Döring, C., Eckerle, S., van Noesel, C. J. M., ... Küppers, R. (2008). Origin and pathogenesis of nodular lymphocyte-predominant Hodgkin lymphoma as revealed by global gene expression analysis. The Journal of experimental medicine, 205(10), 2251-2268. doi:10.1084/jem.20080809

Chang, K. P., Wu, C. C., Fang, K. H., Tsai, C. Y., Chang, Y. L., Liu, S. C., \& Kao, H. K. (2013). Serum levels of chemokine (C-X-C motif) ligand 9 (CXCL9) are associated with tumor progression and treatment outcome in patients with oral cavity squamous cell carcinoma. Oral Oncol, 49(8), 802-807. doi:10.1016/j.oraloncology.2013.05.006

Chin, C.-H., Chen, S.-H., Wu, H.-H., Ho, C.-W., Ko, M.-T., \& Lin, C.-Y. (2014). cytoHubba: identifying hub objects and sub-networks from complex interactome. BMC systems biology, 8 Suppl 4(Suppl 4), S11-S11. doi:10.1186/1752-0509-8-S4-S11

Choesmel, V., Fribourg, S., Aguissa-Touré, A. H., Pinaud, N., Legrand, P., Gazda, H. T., \& Gleizes, P. E. (2008). Mutation of ribosomal protein RPS24 in Diamond-Blackfan anemia results in a ribosome biogenesis disorder. Hum Mol Genet, 17(9), 1253-1263. doi:10.1093/hmg/ddn015

Dancsok, A. R., Gao, D., Lee, A. F., Steigen, S. E., Blay, J. Y., Thomas, D. M., . . Demicco, E. G. (2020). Tumor-associated macrophages and macrophage-related immune checkpoint expression in sarcomas. Oncoimmunology, 9(1), 1747340. doi:10.1080/2162402x.2020.1747340 
513

514

515

516

517

518

519

520

521

522

523

524

525

526

527

528

529

530

531

532

533

534

535

536

537

538

539

540

541

542

543

544

545

546

547

548

549

550

551

552

553

554

555

556

557

558

De Bortoli, M., Castellino, R. C., Lu, X. Y., Deyo, J., Sturla, L. M., Adesina, A. M., . . Kim, J. Y. (2006). Medulloblastoma outcome is adversely associated with overexpression of EEF1D, RPL30, and RPS20 on the long arm of chromosome 8. BMC Cancer, 6, 223. doi:10.1186/1471-2407-6-223

De la Fuente López, M., Landskron, G., Parada, D., Dubois-Camacho, K., Simian, D., Martinez, M., .. . Hermoso, R. M. (2018). The relationship between chemokines CCL2, CCL3, and CCL4 with the tumor microenvironment and tumor-associated macrophage markers in colorectal cancer. Tumour Biol, 4O(11), 1010428318810059. doi:10.1177/1010428318810059

Fedoriw, Y., Selitsky, S., Montgomery, N. D., Kendall, S. M., Richards, K. L., Du, W., . . Gopal, S. (2020). Identifying transcriptional profiles and evaluating prognostic biomarkers of HIV-associated diffuse large B-cell lymphoma from Malawi. Mod Pathol, 33(8), 1482-1491. doi:10.1038/s41379020-0506-3

Gandhi, M. K., Hoang, T., Law, S. C., Brosda, S., O'Rourke, K., Tobin, J. W. D., . . Keane, C. (2020). EBVtissue positive primary CNS lymphoma occurring after immunosuppression is a distinct immunobiological entity. Blood. doi:10.1182/blood.2020008520

Gautier, L., Cope, L., Bolstad, B. M., \& Irizarry, R. A. (2004). affy-analysis of Affymetrix GeneChip data at the probe level. Bioinformatics, 20(3), 307-315. doi:10.1093/bioinformatics/btg405

He, W., Chen, L., Chen, L., Yuan, G., Fang, Y., Chen, W., . . Gale, R. P. (2020). COVID-19 in persons with haematological cancers. Leukemia, 34(6), 1637-1645. doi:10.1038/s41375-020-0836-7

Huang da, W., Sherman, B. T., \& Lempicki, R. A. (2009). Systematic and integrative analysis of large gene lists using DAVID bioinformatics resources. Nat Protoc, 4(1), 44-57. doi:10.1038/nprot.2008.211

Huang, Q., Liu, F., \& Shen, J. (2019). Bioinformatic validation identifies candidate key genes in diffuse large-B cell lymphoma. Per Med, 16(4), 313-323. doi:10.2217/pme-2018-0068

Karube, K., Enjuanes, A., Dlouhy, I., Jares, P., Martin-Garcia, D., Nadeu, F., . . Campo, E. (2018). Integrating genomic alterations in diffuse large B-cell lymphoma identifies new relevant pathways and potential therapeutic targets. Leukemia, 32(3), 675-684. doi:10.1038/leu.2017.251

Kawahata, T., Kawahara, K., Shimokawa, M., Sakiyama, A., Shiraishi, T., Minami, K., . . Furukawa, T. (2020). Involvement of ribosomal protein L11 expression in sensitivity of gastric cancer against 5-FU. Oncol Lett, 19(3), 2258-2264. doi:10.3892/ol.2020.11352

Kayama, K., Watanabe, S., Takafuji, T., Tsuji, T., Hironaka, K., Matsumoto, M., . . Fujita, M. (2017). GRWD1 negatively regulates p53 via the RPL11-MDM2 pathway and promotes tumorigenesis. EMBO Rep, 18(1), 123-137. doi:10.15252/embr.201642444

Langfelder, P., \& Horvath, S. (2008). WGCNA: an R package for weighted correlation network analysis. BMC Bioinformatics, 9(1), 559. doi:10.1186/1471-2105-9-559

Langfelder, P., \& Horvath, S. (2012). Fast R Functions for Robust Correlations and Hierarchical Clustering. J Stat Softw, 46(11).

Lee, L. Y. W., Cazier, J. B., Starkey, T., Briggs, S. E. W., Arnold, R., Bisht, V., . . Kerr, R. (2020). COVID-19 prevalence and mortality in patients with cancer and the effect of primary tumour subtype and patient demographics: a prospective cohort study. Lancet Oncol, 21(10), 1309-1316. doi:10.1016/s1470-2045(20)30442-3

Liang, Z., Mou, Q., Pan, Z., Zhang, Q., Gao, G., Cao, Y., . . Feng, W. (2019). Identification of candidate diagnostic and prognostic biomarkers for human prostate cancer: RPL22L1 and RPS21. Med Oncol, 36(6), 56. doi:10.1007/s12032-019-1283-z

Liao, M., Liu, Y., Yuan, J., Wen, Y., Xu, G., Zhao, J., . . Zhang, Z. (2020). Single-cell landscape of bronchoalveolar immune cells in patients with COVID-19. Nat Med, 26(6), 842-844. doi:10.1038/s41591-020-0901-9

Peer) reviewing PDF | (2021:06:62901:2:0:NEW 30 Sep 2021) 
559

560

561

562

563

564

565

566

567

568

569

570

571

572

573

574

575

576

577

578

579

580

581

582

583

584

585

586

587

588

589

590

591

592

593

594

595

596

597

598

599

600

601

602

603

604

605

606
Liu, B. B., Ma, T., Sun, W., Gao, W. Y., Liu, J. M., Li, L. Q., ... Guo, Y. Y. (2021). Centromere protein U enhances the progression of bladder cancer by promoting mitochondrial ribosomal protein $\mathrm{s} 28$ expression. Korean J Physiol Pharmacol, 25(2), 119-129. doi:10.4196/kjpp.2021.25.2.119

Liu, J., Zhou, S., Li, S., Jiang, Y., Wan, Y., Ma, X., \& Cheng, W. (2019). Eleven genes associated with progression and prognosis of endometrial cancer (EC) identified by comprehensive bioinformatics analysis. Cancer cell international, 19, 136-136. doi:10.1186/s12935-019-0859-1

Liu, W., Liu, J., Song, Y., Zeng, X., Wang, X., Mi, L., . . . Union for China Lymphoma Investigators of the Chinese Society of Clinical, O. (2019). Burden of lymphoma in China, 2006-2016: an analysis of the Global Burden of Disease Study 2016. Journal of hematology \& oncology, 12(1), 115-115. doi:10.1186/s13045-019-0785-7

Lui, T. W. H., Tsui, N. B. Y., Chan, L. W. C., Wong, C. S. C., Siu, P. M. F., \& Yung, B. Y. M. (2015). DECODE: an integrated differential co-expression and differential expression analysis of gene expression data. BMC Bioinformatics, 16(1), 182. doi:10.1186/s12859-015-0582-4

Luo, B., Gu, Y.-y., Wang, X.-d., Chen, G., \& Peng, Z.-g. (2018). Identification of potential drugs for diffuse large b-cell lymphoma based on bioinformatics and Connectivity Map database. Pathology Research and Practice, 214(11), 1854-1867. doi:https://doi.org/10.1016/i.prp.2018.09.013

Martínez-Reyes, I., Cardona, L. R., Kong, H., Vasan, K., McElroy, G. S., Werner, M., . . Chandel, N. S. (2020). Mitochondrial ubiquinol oxidation is necessary for tumour growth. Nature, 585(7824), 288-292. doi:10.1038/s41586-020-2475-6

Naresh, K. N., Raphael, M., Ayers, L., Hurwitz, N., Calbi, V., Rogena, E., . . Leoncini, L. (2011). Lymphomas in sub-Saharan Africa--what can we learn and how can we help in improving diagnosis, managing patients and fostering translational research? Br J Haematol, 154(6), 696703. doi:10.1111/j.1365-2141.2011.08772.x

Newman, A. M., Steen, C. B., Liu, C. L., Gentles, A. J., Chaudhuri, A. A., Scherer, F., . . Alizadeh, A. A. (2019). Determining cell type abundance and expression from bulk tissues with digital cytometry. Nature Biotechnology, 37(7), 773-782. doi:10.1038/s41587-019-0114-2

Norberg, E., Lako, A., Chen, P. H., Stanley, I. A., Zhou, F., Ficarro, S. B., . . Danial, N. N. (2017). Differential contribution of the mitochondrial translation pathway to the survival of diffuse large B-cell lymphoma subsets. Cell Death Differ, 24(2), 251-262. doi:10.1038/cdd.2016.116

Pasqualucci, L., \& Dalla-Favera, R. (2018). Genetics of diffuse large B-cell lymphoma. Blood, 131(21), 2307-2319. doi:10.1182/blood-2017-11-764332

Passamonti, F., Cattaneo, C., Arcaini, L., Bruna, R., Cavo, M., Merli, F., . . Corradini, P. (2020). Clinical characteristics and risk factors associated with COVID-19 severity in patients with haematological malignancies in Italy: a retrospective, multicentre, cohort study. Lancet Haematol, 7(10), e737-e745. doi:10.1016/s2352-3026(20)30251-9

Rhodes, D. R., Yu, J., Shanker, K., Deshpande, N., Varambally, R., Ghosh, D., . . Chinnaiyan, A. M. (2004). ONCOMINE: a cancer microarray database and integrated data-mining platform. Neoplasia, 6(1), 1-6. doi:10.1016/s1476-5586(04)80047-2

Ritchie, M. E., Phipson, B., Wu, D., Hu, Y., Law, C. W., Shi, W., \& Smyth, G. K. (2015). limma powers differential expression analyses for RNA-sequencing and microarray studies. Nucleic Acids Research, 43(7), e47-e47. doi:10.1093/nar/gkv007

Shah, V., Ko Ko, T., Zuckerman, M., Vidler, J., Sharif, S., Mehra, V., . . Kulasekararaj, A. G. (2020). Poor outcome and prolonged persistence of SARS-CoV-2 RNA in COVID-19 patients with haematological malignancies; King's College Hospital experience. Br J Haematol, 190(5), e279e282. doi:10.1111/bjh.16935

Shannon, P., Markiel, A., Ozier, O., Baliga, N. S., Wang, J. T., Ramage, D., . . Ideker, T. (2003). Cytoscape: a software environment for integrated models of biomolecular interaction networks. Genome research, 13(11), 2498-2504. doi:10.1101/gr.1239303

Peer) reviewing PDF | (2021:06:62901:2:0:NEW 30 Sep 2021) 
607

608

609

610

611

612

613

614

615

616

617

618

619

620

621

622

623

624

625

626

627

628

629

630

631

632

633

634

635

636

637

638

639

640

641

642

643

644

645

646

647

648

649

650

651

652

Stelzer, G., Rosen, N., Plaschkes, I., Zimmerman, S., Twik, M., Fishilevich, S., . . Lancet, D. (2016). The GeneCards Suite: From Gene Data Mining to Disease Genome Sequence Analyses. Curr Protoc Bioinformatics, 54, 1.30.31-31.30.33. doi:10.1002/cpbi.5

Subramanian, A., Tamayo, P., Mootha, V. K., Mukherjee, S., Ebert, B. L., Gillette, M. A., . . Mesirov, J. P. (2005). Gene set enrichment analysis: A knowledge-based approach for interpreting genomewide expression profiles. Proceedings of the National Academy of Sciences, 102(43), 15545. doi:10.1073/pnas.0506580102

Szklarczyk, D., Gable, A. L., Lyon, D., Junge, A., Wyder, S., Huerta-Cepas, J., . . Mering, C. V. (2019). STRING v11: protein-protein association networks with increased coverage, supporting functional discovery in genome-wide experimental datasets. Nucleic Acids Res, 47(D1), D607d613. doi:10.1093/nar/gky1131

Tang, Z., Kang, B., Li, C., Chen, T., \& Zhang, Z. (2019). GEPIA2: an enhanced web server for large-scale expression profiling and interactive analysis. Nucleic Acids Res, 47(W1), W556-w560. doi:10.1093/nar/gkz430

Team, R. C. (2019). R: A language and environment for statistical computing. R Foundation for Statistical Computing, Vienna, Austria. URL https://www.R-project.org/.

Uhlen, M., Zhang, C., Lee, S., Sjöstedt, E., Fagerberg, L., Bidkhori, G., . . Ponten, F. (2017). A pathology atlas of the human cancer transcriptome. Science, 357(6352), eaan2507. doi:10.1126/science.aan2507

van Dam, S., Võsa, U., van der Graaf, A., Franke, L., \& de Magalhães, J. P. (2018). Gene co-expression analysis for functional classification and gene-disease predictions. Briefings in bioinformatics, 19(4), 575-592. doi:10.1093/bib/bbw139

Visco, C., Li, Y., Xu-Monette, Z. Y., Miranda, R. N., Green, T. M., Li, Y., . . Young, K. H. (2012). Comprehensive gene expression profiling and immunohistochemical studies support application of immunophenotypic algorithm for molecular subtype classification in diffuse large B-cell lymphoma: a report from the International DLBCL Rituximab-CHOP Consortium Program Study. Leukemia, 26(9), 2103-2113. doi:10.1038/leu.2012.83

Wang, Y., Sui, J., Li, X., Cao, F., He, J., Yang, B., . . Pu, Y. D. (2015). RPS24 knockdown inhibits colorectal cancer cell migration and proliferation in vitro. Gene, 571(2), 286-291. doi:10.1016/j.gene.2015.06.084

Wickham, H. (2016). ggplot2: Elegant Graphics for Data Analysis. Springer-Verlag New York, ISBN 978973-319-24277-24274.

Xiao, J., Wang, X., \& Bai, H. (2020). Clinical Features and Prognostic Impact of Coexpression Modules Constructed by WGCNA for Diffuse Large B-Cell Lymphoma. BioMed research international, 2020, 7947208. doi:10.1155/2020/7947208

Yi, J. H., Yoon, S. E., Ryu, K. J., Ko, Y. H., Kim, W. S., \& Kim, S. J. (2020). Pre-treatment serum IL-10 predicts the risk of secondary central nervous system involvement in patients with diffuse large B-cell lymphoma. Cytokine, 129, 155048. doi:10.1016/j.cyto.2020.155048

Yin, Z., Bai, L., Li, W., Zeng, T., Tian, H., \& Cui, J. (2019). Targeting T cell metabolism in the tumor microenvironment: an anti-cancer therapeutic strategy. Journal of Experimental \& Clinical Cancer Research, 38(1), 403. doi:10.1186/s13046-019-1409-3

Yu, G., Wang, L. G., Han, Y., \& He, Q. Y. (2012). clusterProfiler: an R package for comparing biological themes among gene clusters. Omics, 16(5), 284-287. doi:10.1089/omi.2011.0118

Zhou, L., Ding, L., Gong, Y., Zhao, J., Xin, G., Zhou, R., \& Zhang, W. (2020). Identification of hub genes associated with the pathogenesis of diffuse large B-cell lymphoma subtype one characterized by host response via integrated bioinformatic analyses. PeerJ, 8, e10269. doi:10.7717/peerj.10269

Peer] reviewing PDF | (2021:06:62901:2:0:NEW 30 Sep 2021) 
653 Zhou, R., Zhang, J., Zeng, D., Sun, H., Rong, X., Shi, M., ... Liao, W. (2019). Immune cell infiltration as a biomarker for the diagnosis and prognosis of stage I-III colon cancer. Cancer immunology,

656

657

658 immunotherapy : CII, 68(3), 433-442. doi:10.1007/s00262-018-2289-7

Zou, D., Zhang, H., Ke, J., Li, J., Zhu, Y., Gong, Y., ... Miao, X. (2020). Three functional variants were identified to affect RPS24 expression and significantly associated with risk of colorectal cancer. Arch Toxicol, 94(1), 295-303. doi:10.1007/s00204-019-02600-9

659

660 


\section{Figure 1}

Statistics for the differentially expressed genes.

(A) Volcano plot highlighting significant genes in DLBCL and non-tumor tissues. UP represents upregulated; DOWN, downregulated; NOT, not significant. (B) Heatmap of the top 169 DEGs between DLBCL cases and controls (NORMAL); $|\log 2 \mathrm{FC}|>2$, $p$-value $<0.05$; the range of the colors corresponds with the range of expression values. (C) Venn diagram shows 118 overlapping (common) DEGs screened using 5 Cytohubba centrality methods. DEGs, differentially expressed genes. 
A

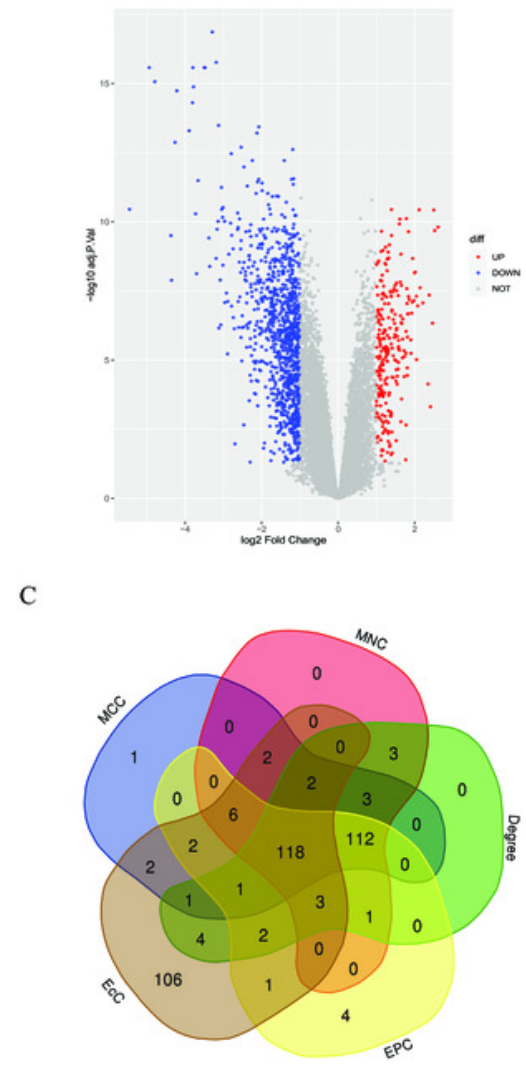

B

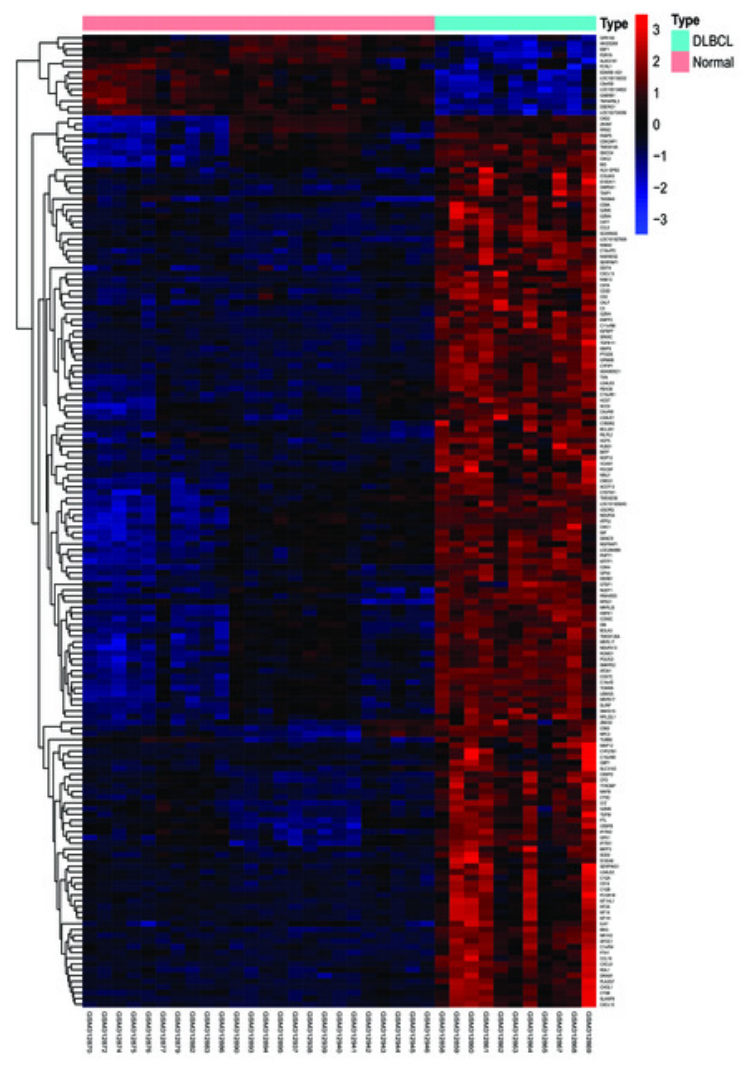




\section{Figure 2}

Gene ontology (GO) and KEGG enrichment analysis of all differentially expressed genes (DEGs).

(A) In GO analysis, the top 10 significantly enriched DEGs. The $x$-axis is the number of DEGs involved in the GO terms; the $y$-axis is the significantly enriched GO terms. (B) In KEGG analysis, the top 15 significantly enriched pathways of the DEGs. KEGG, Kyoto encyclopedia of genes and genomes.

A

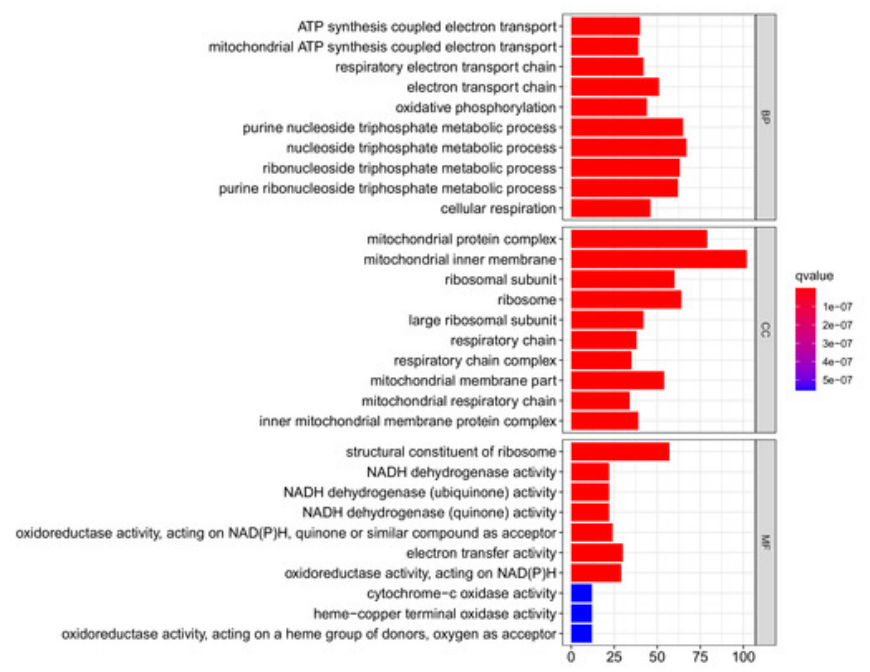

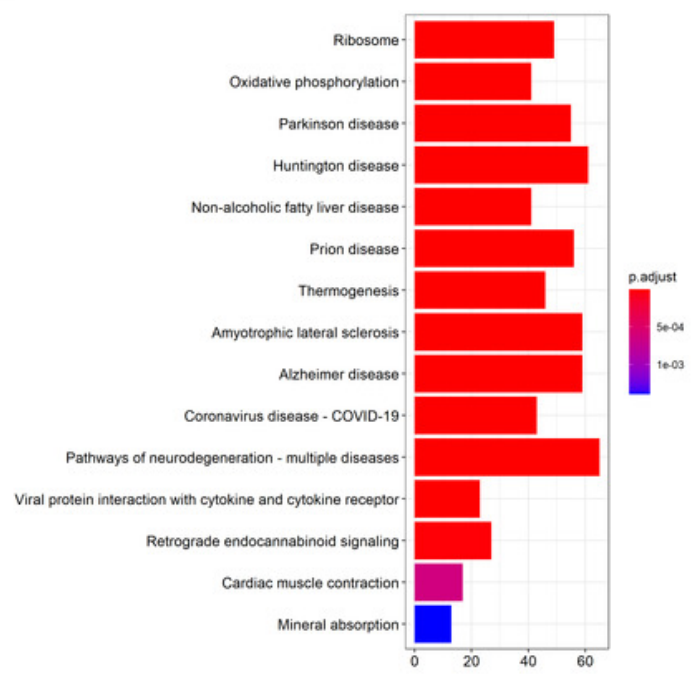




\section{Figure 3}

The prediction of tumor-infiltrating immune cells (TIICs) using the GSE12453 dataset.

Violin plot comparing the proportions of TIICs between non-tumor controls (in blue ) and DLBCL (in red). The $x$ and $y$ axes represent TIICs and their relative percentages, respectively. There was no T cell CD4 memory resting. (B) Bar plots for 24 non-tumor controls and 11 DLBCL samples (x-axis) and the percentages of immune cell subsets (y-axis).

A

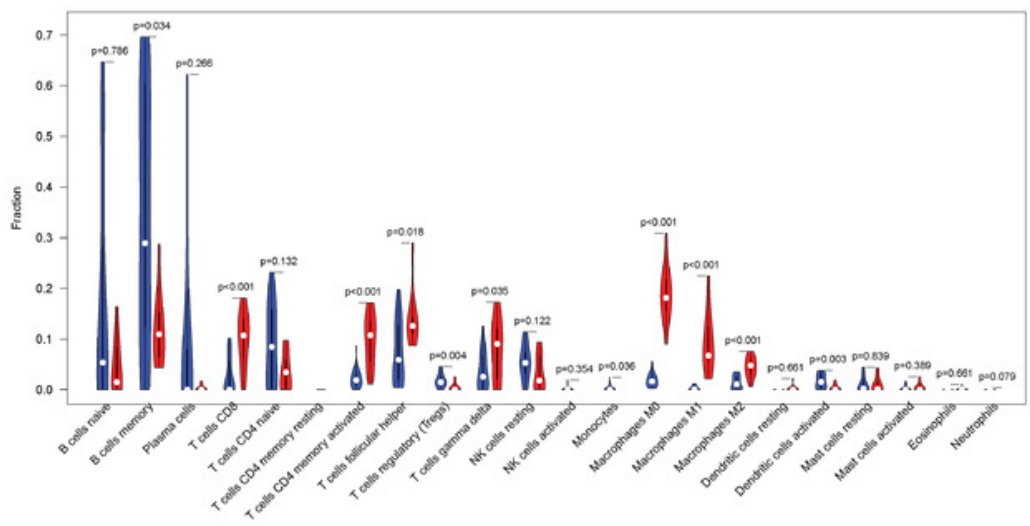

B

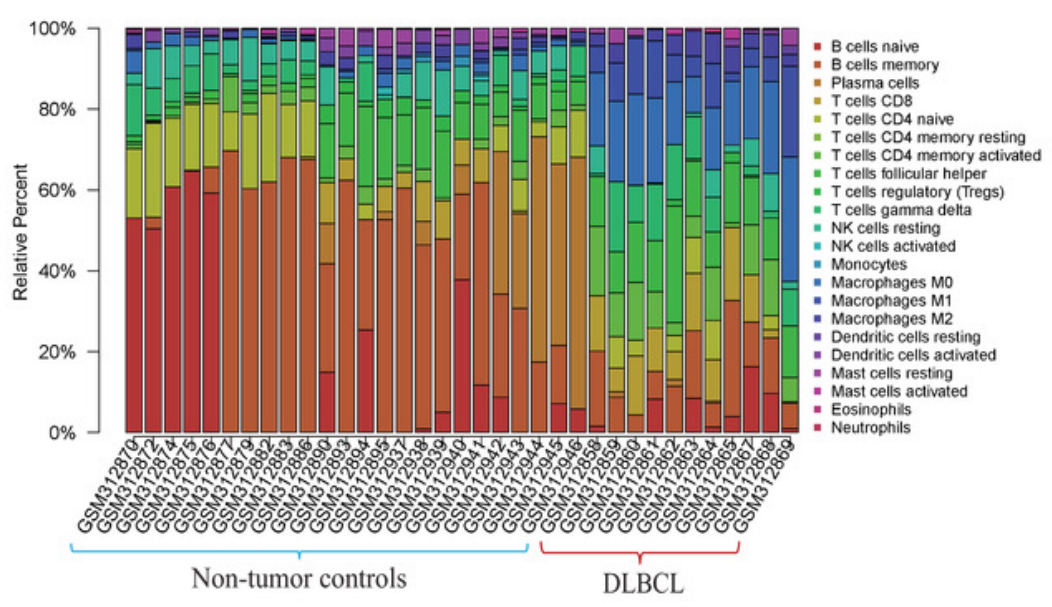


Figure 4

The protein-protein interaction (PPI) networks using the STRING and Cytoscape databases.

(A) The PPI network for the 109 highly connected DEGs; confidence score, 0.9. (B) Cluster 1 consisted of 61 nodes, 995 edges with the highest k-score of 33.17. (C) Cluster 2 had 28 nodes, 359 edges, and a k-score of 26.59. (D) Cluster 3 had 20 nodes, 190 edges, and a kscore of 20.00. (e) The top 250 ranked DEGs. 
A
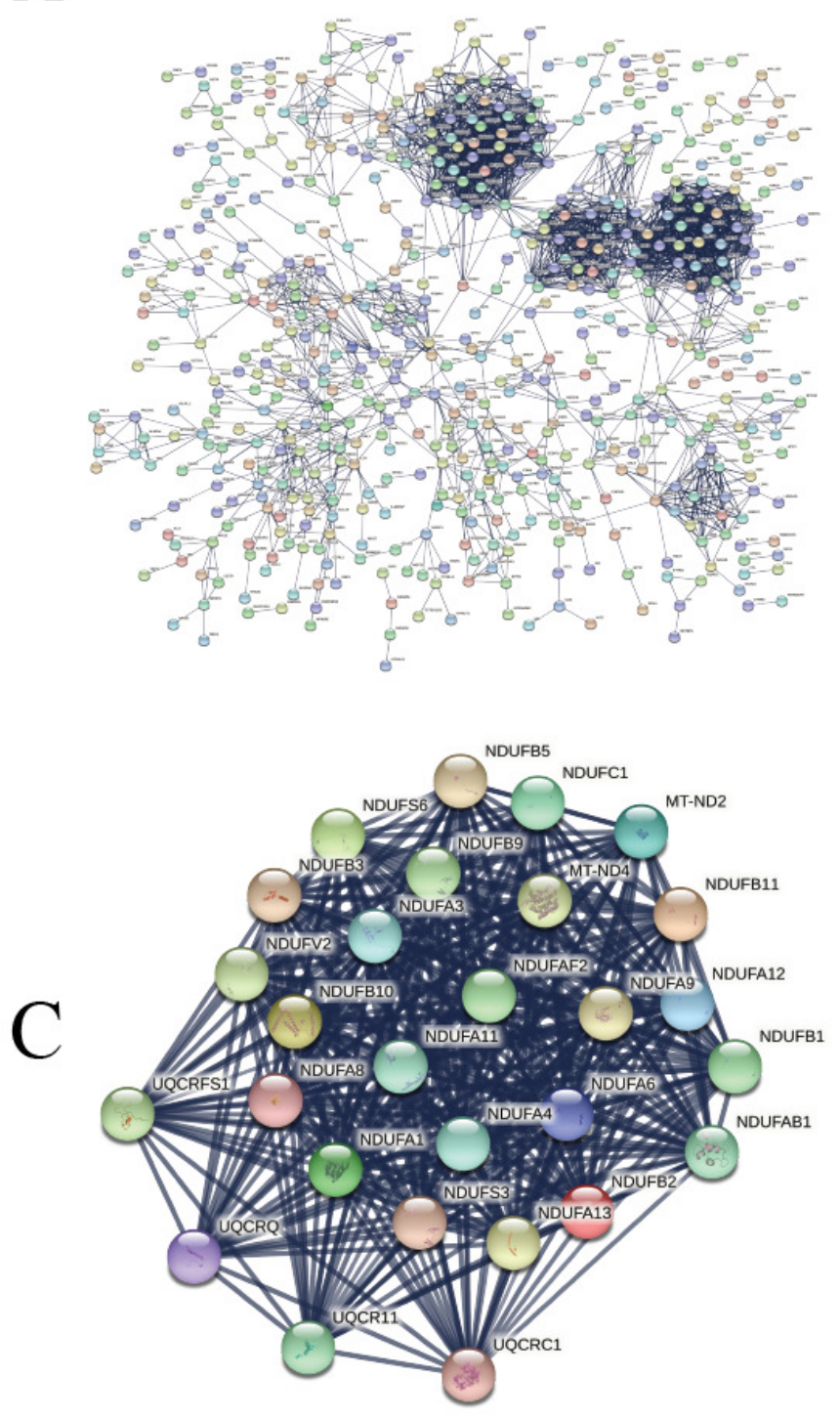

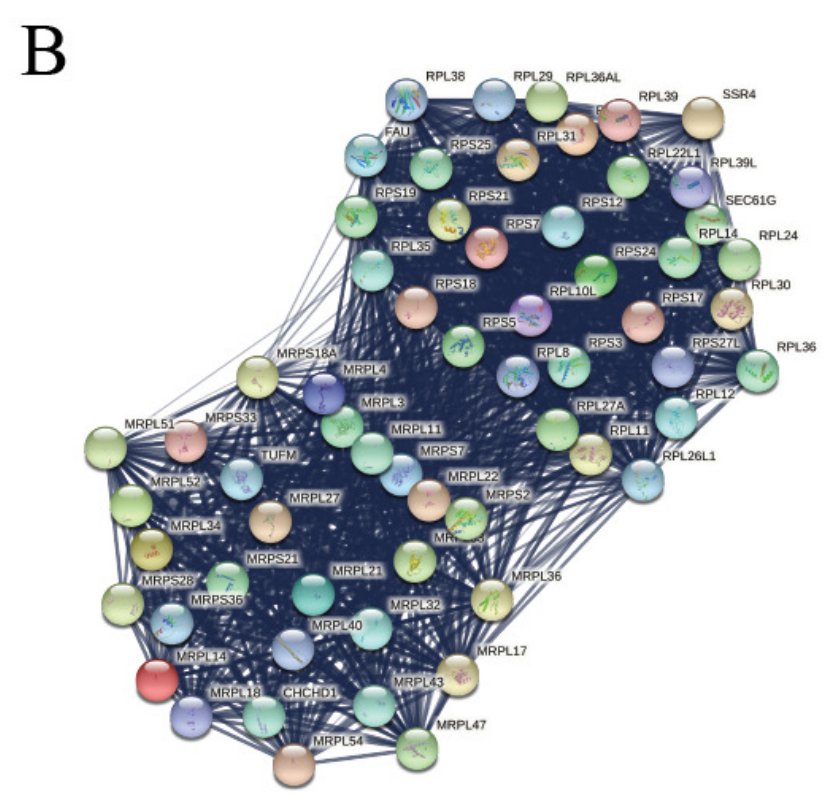

D

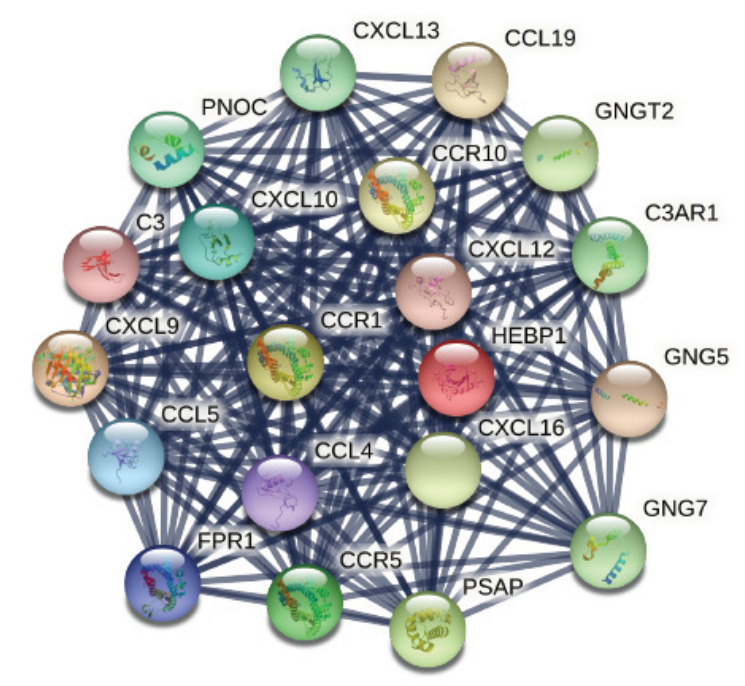

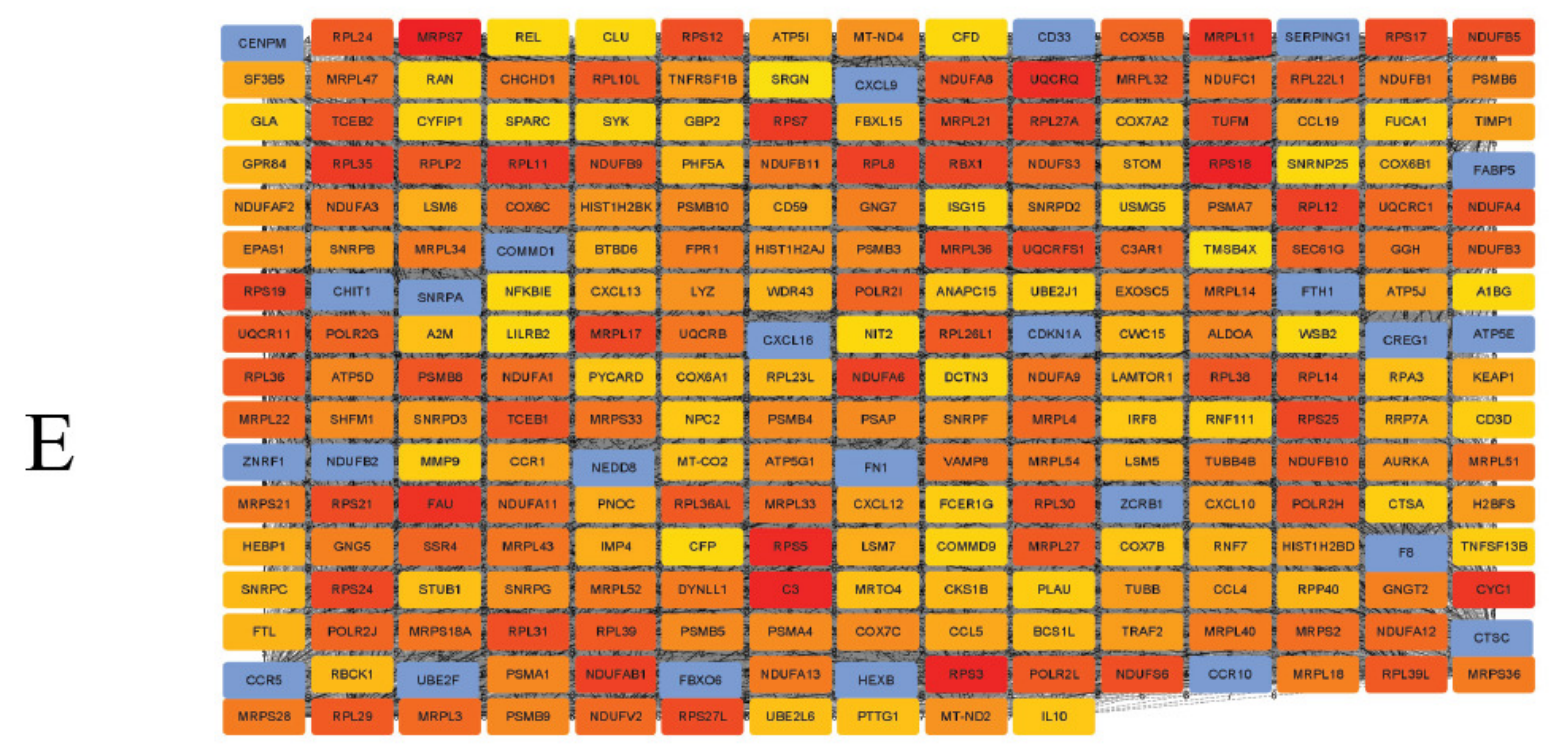




\section{Figure 5}

Construction of gene co-expression network.

(A) Sample clustering to detect outliers, no obvious outliers. (B and C) Determination of softthreshold power. When $\beta$ is set at 8 , the log-log plot of the network connectivity distribution produces a straight line. (D) Hierarchical clustering dendrograms (top modules). Each color band (bottom) represents a color-coded module that contains a group of highly connected genes. The Dynamic Tree Cut identified 18 modules. (E) A heatmap showing the topological overlap matrix (TOM) among the top 1000 genes selected from all genes. The color intensity indicates the correlation strength between pairs of modules: the left side (gene dendrogram) and the top side (module assignment). 
A

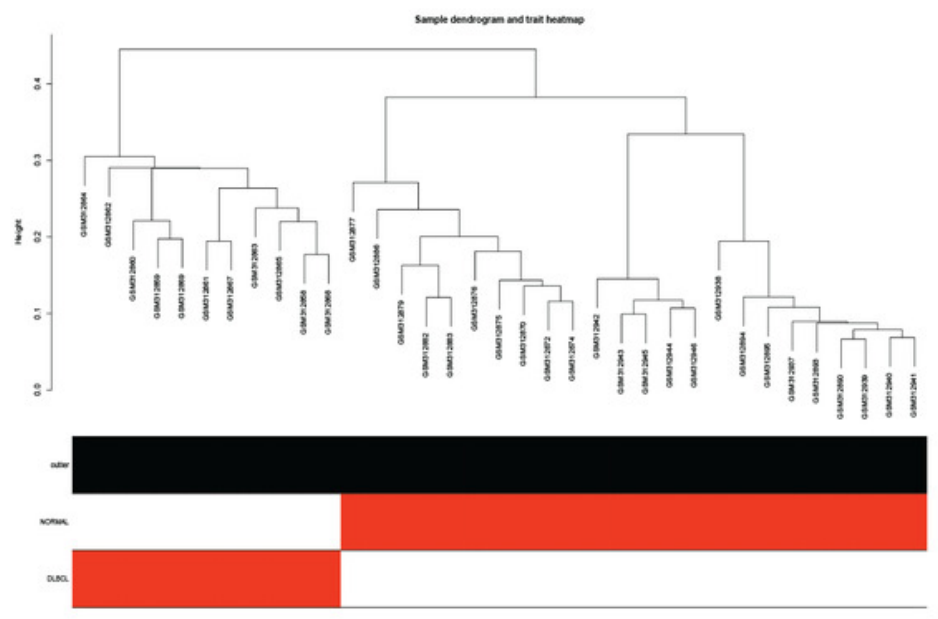

C
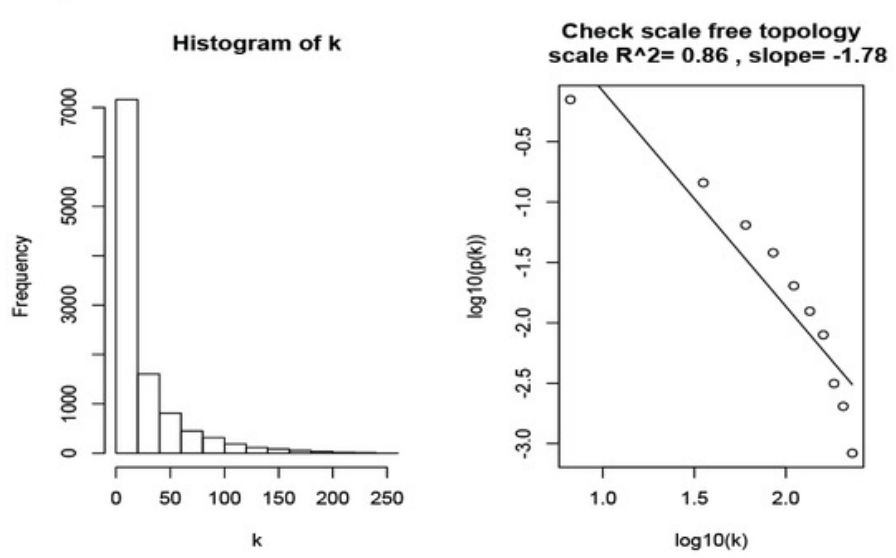

B

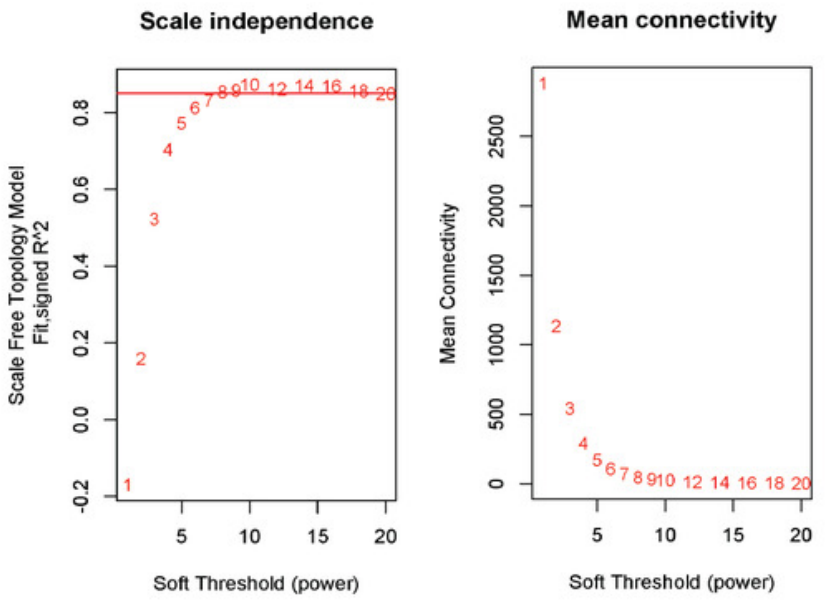

D

E

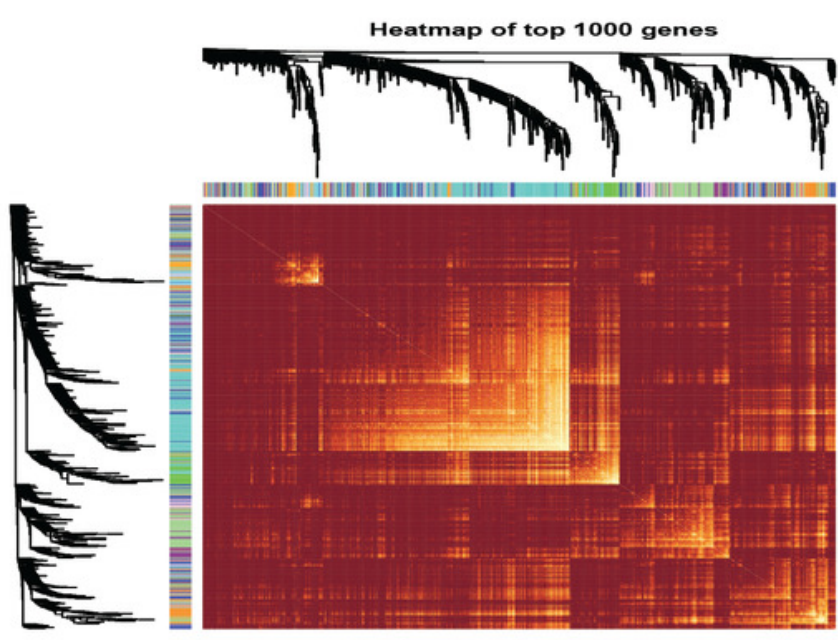




\section{Figure 6}

Identifying modules of clinical relevance from the GSE12453 dataset.

(A) Module trait relationship showing correlation coefficients between module eigengenes (row) and disease status (column), with the corresponding p-values in brackets. The degree of correlation is based on a color legend: red, strong positive and blue, strong negative correlation. (B) Heatmap plot of the adjacencies in the eigengene network, including the relationship with DLBCL trait. The top panel is the hierarchical clustering dendrogram of the eigengenes. The bottom panel shows the eigengene adjacency. (C) Scatter plots of gene significance (GS) versus module membership (MM) for the DLBCL related modules (turquoise, green and dark magenta, respectively). 
A

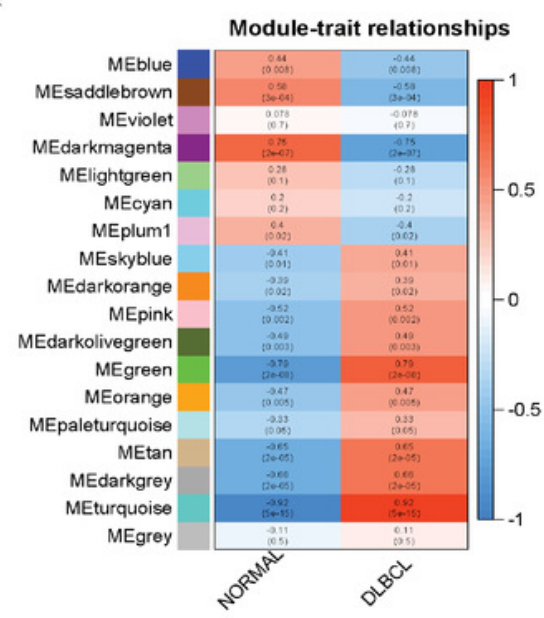

B
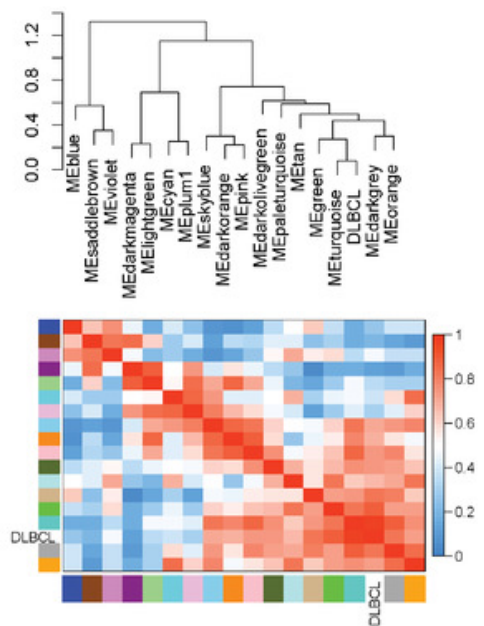

C
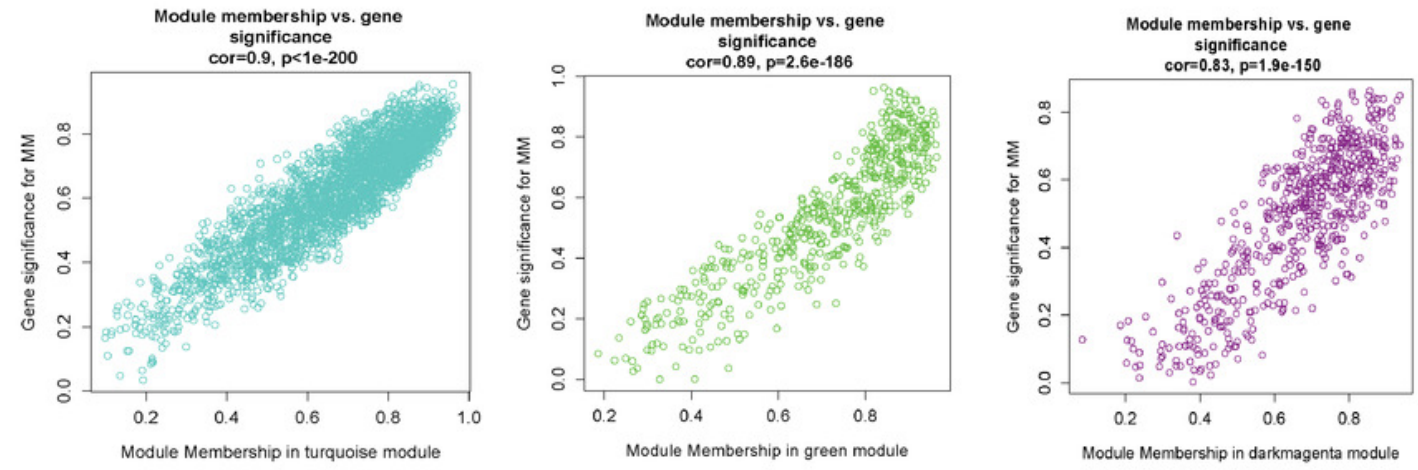
Figure 7

Top 10 enrichment results of the 195 WGCNA (weighted correlation network analysis) genes.

(A) Gene ontology (GO) functional analysis. (B) Kyoto encyclopedia of genes and genomes (KEGG) pathway analysis.

A

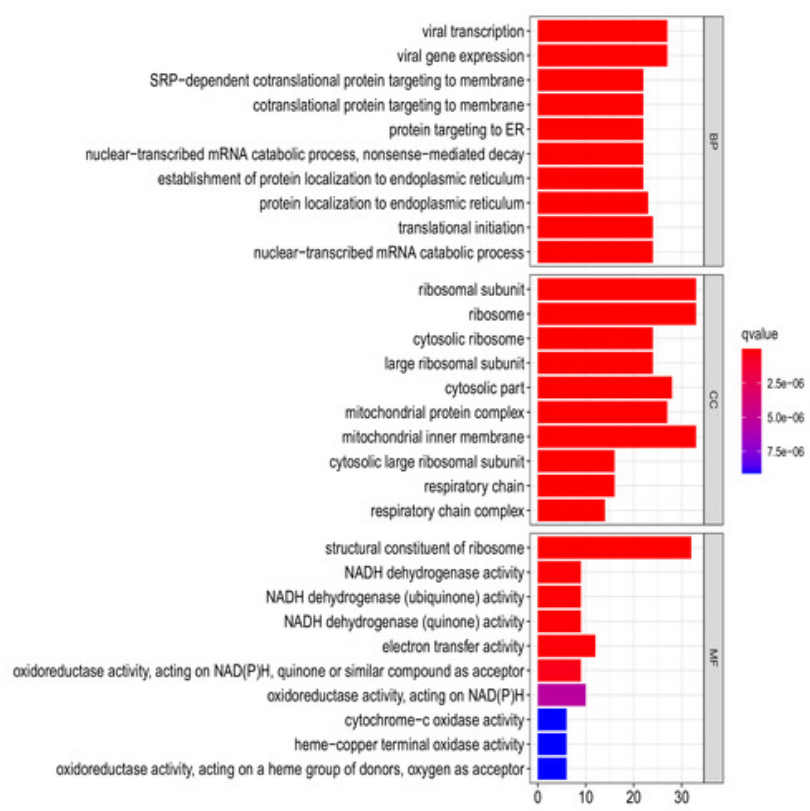

B

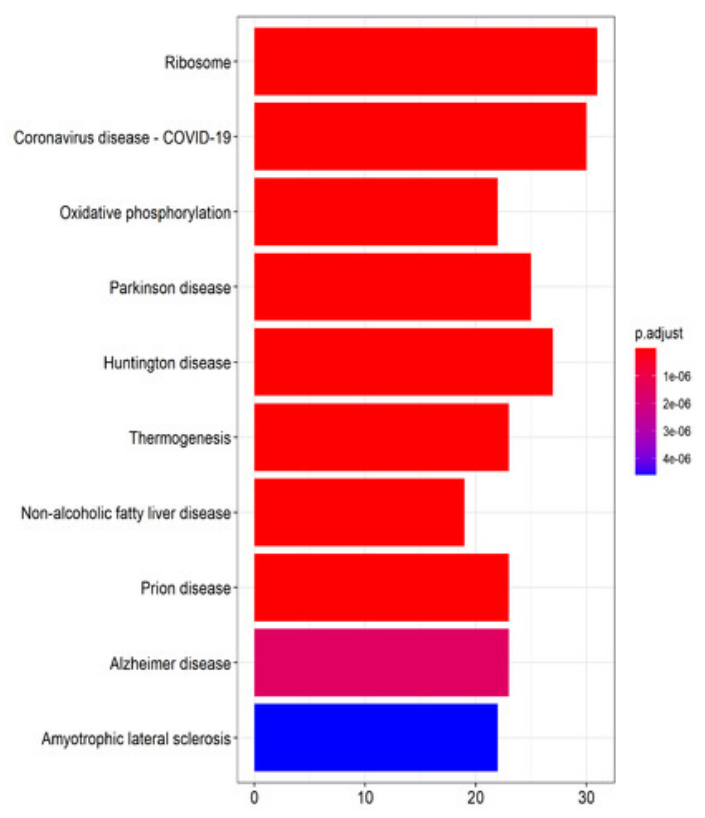




\section{Figure 8}

Differentially co-expressed hub genes.

(A) Venn diagram indicating the 46 common genes. (B) Protein-protein interaction (PPI) network of the 46 hub genes (STRING and Cytoscape databases).

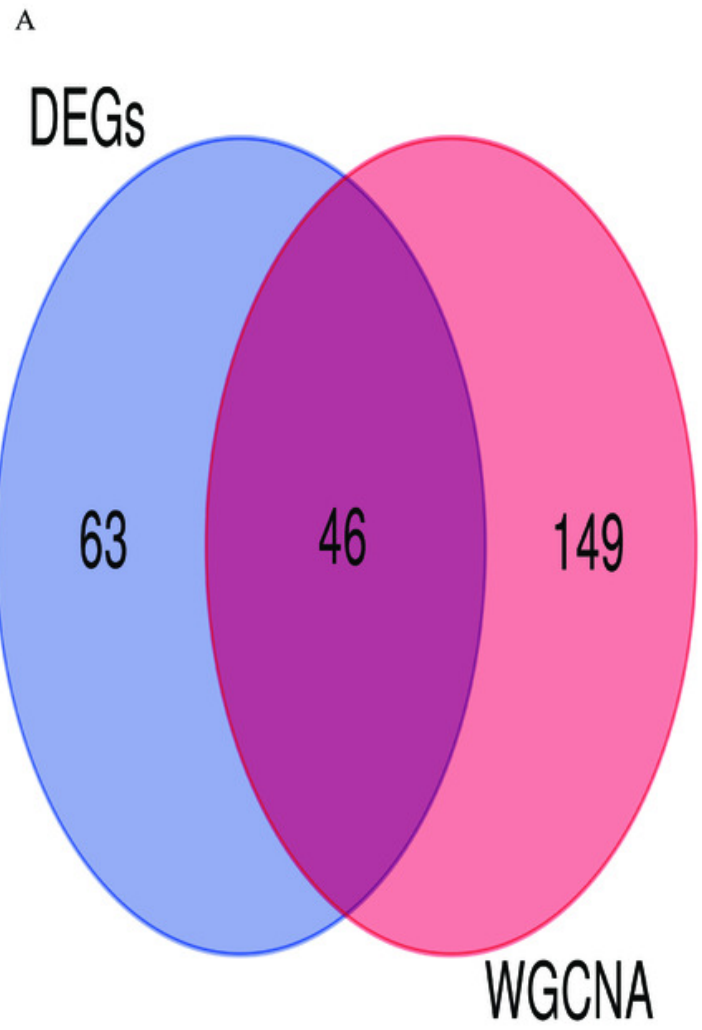

B

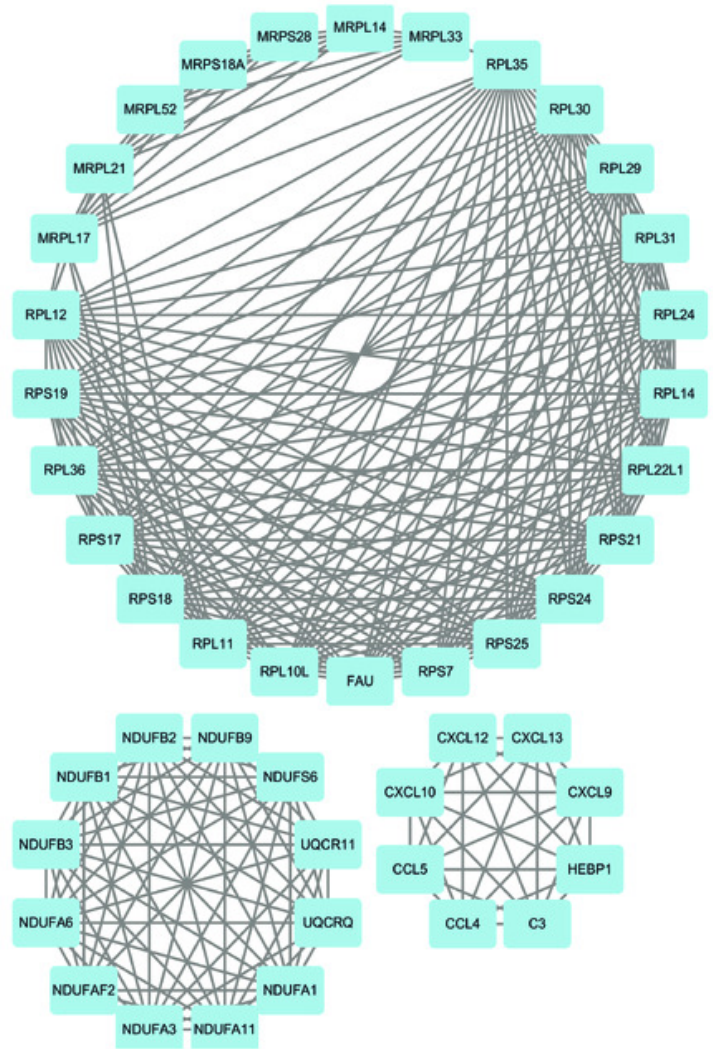


Figure 9

The expressions of the hub genes in the GEPIA2 database.

$*(p<0.01)$. The data were retrieved from the GEPIA 2 database

(http://gepia2.cancer-pku.cn/\#index).
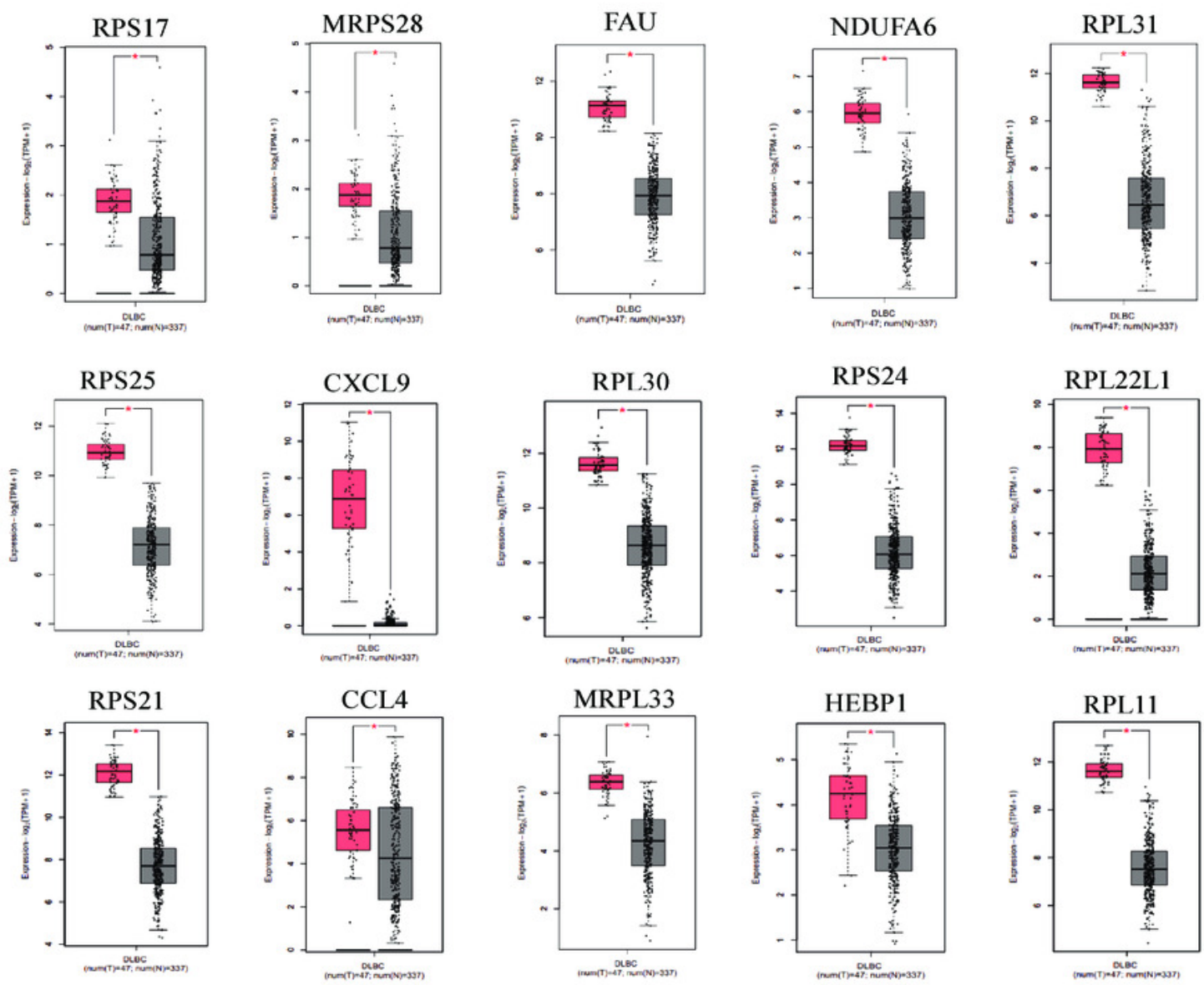
Figure 10

The Kaplan-Meier estimates for the overall survival (OS) of the 15 core hub genes in GSE31312 ( $p<0.05)$.

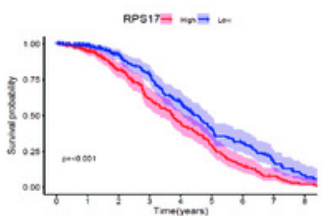

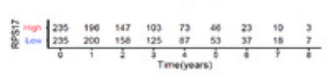

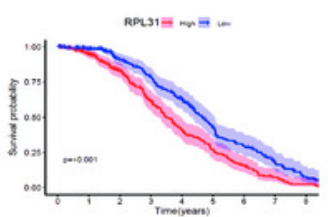

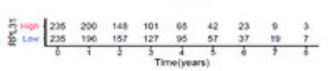

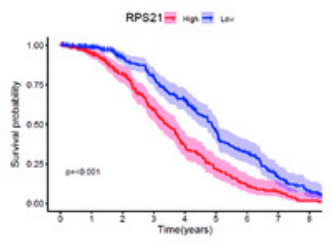

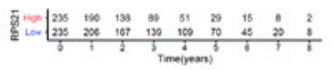

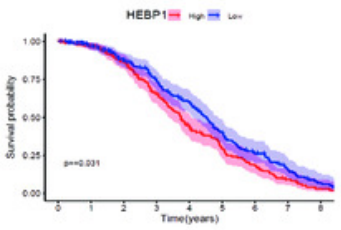

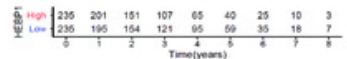
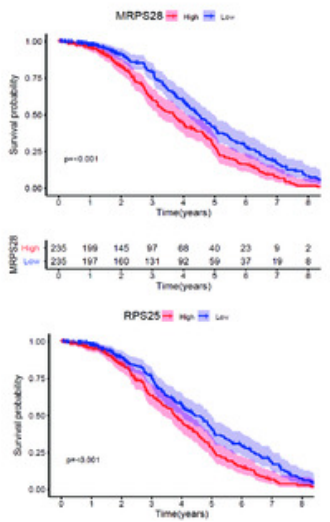

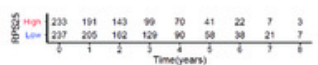

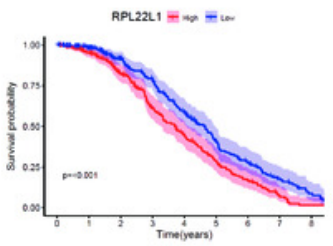

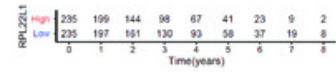

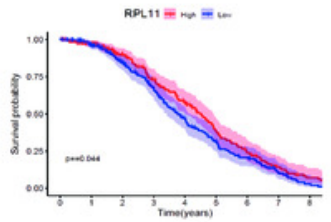

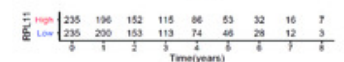
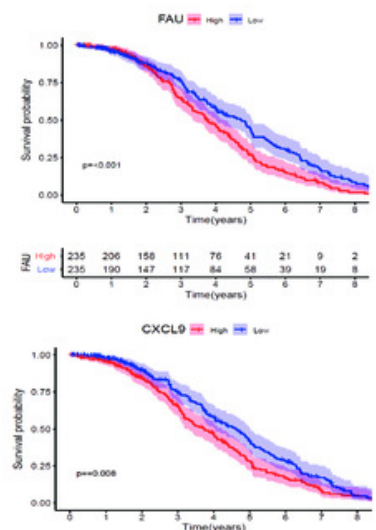

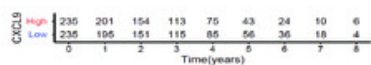

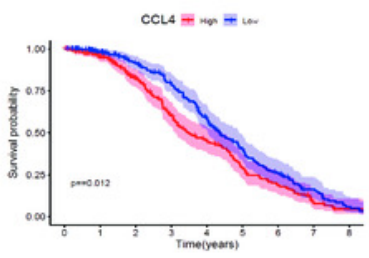

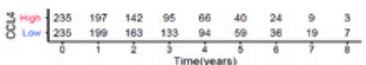

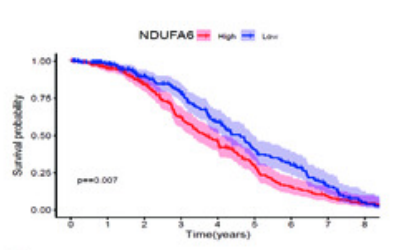

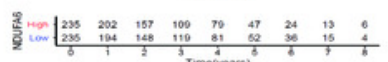

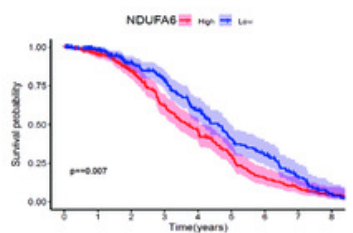

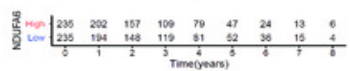

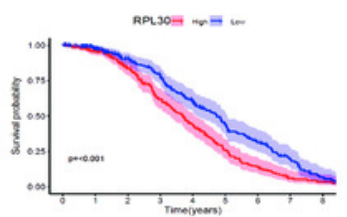

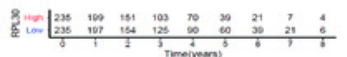

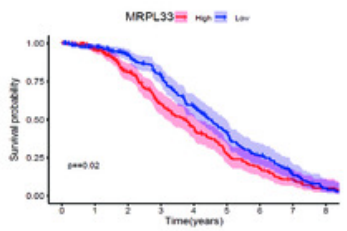

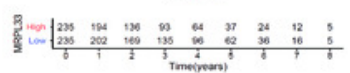

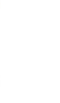

\footnotetext{
(1)
}


Figure 11

PPI and enrichment analysis of the 15 core hub genes.

(A) Cluster analysis. Genes in the circle represent covid 19 pathway genes. (B) The top 5 gene ontology (GO) terms. (C) The top 3 KEGG (Kyoto encyclopedia of genes and genomes) pathways.

A
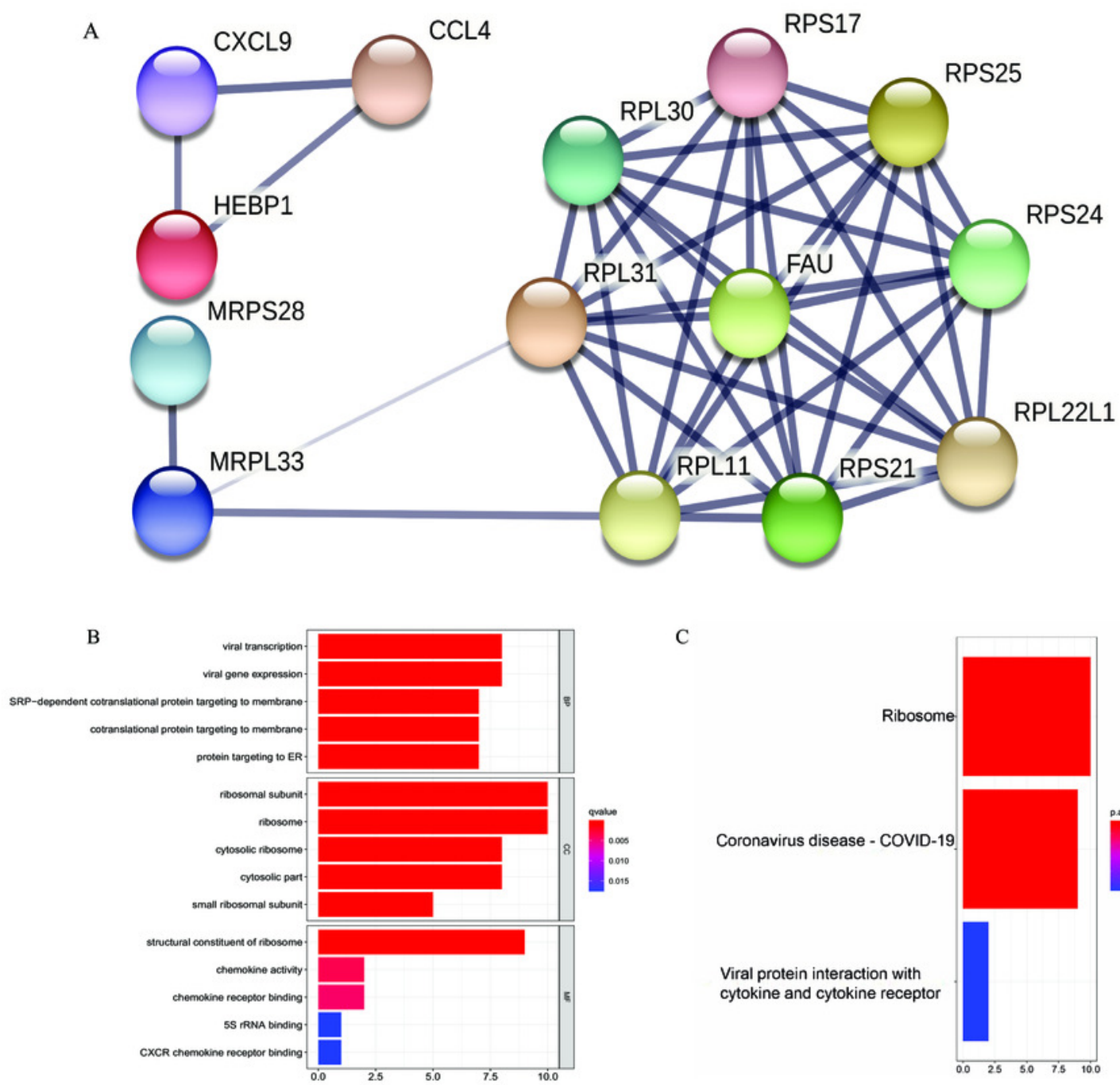

$\mathrm{C}$

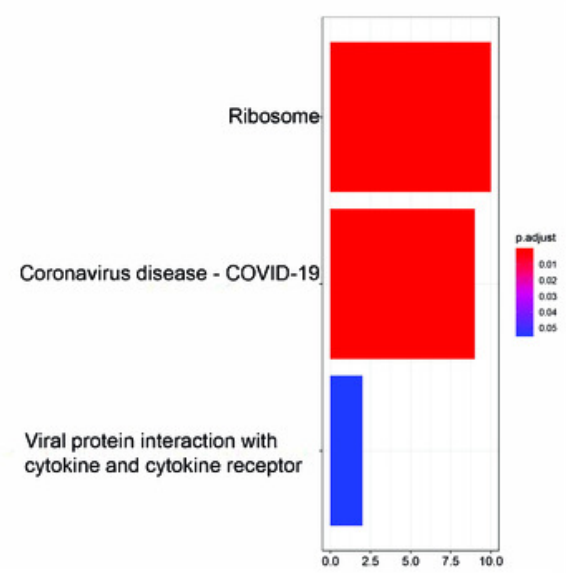




\section{Table $\mathbf{1}$ (on next page)}

Gene ontology and KEGG pathway analysis of the 46 differentially co-expressed genes.

Selection of terms enriched in the categories based on the most significant adjusted $p$-value (p. adjust). Count: the number of genes enriched in each term or pathway. 


\section{Biological process}

GO:0006614 SRP-dependent cotranslational protein targeting $\quad 16 \quad 1.29 \mathrm{E}-22$ to membrane

GO:0019083 Viral transcription $\quad \begin{array}{lll}18 & 1.29 \mathrm{E}-22\end{array}$

GO:0006613 Cotranslational protein targeting to membrane $\quad 16 \quad 1.29 \mathrm{E}-22$

GO:0019080 Viral gene expression $\quad 18 \quad 2.85 \mathrm{E}-22$

GO:0045047 Protein targeting to ER $\quad \begin{array}{lll}16 & 2.98 \mathrm{E}-22\end{array}$

GO:0000184 Nuclear-transcribed mRNA catabolic process, $16 \quad 3.30 \mathrm{E}-22$ nonsense-mediated decay

GO:0072599 Establishment of protein localization to $16 \quad 3.74 \mathrm{E}-22$ endoplasmic reticulum

GO:0070972 Protein localization to endoplasmic reticulum $\quad 16 \quad 7.45 \mathrm{E}-21$

\section{Cellular component}

GO:0044391 Ribosomal subunit

GO:0005840 Ribosome

$25 \quad 1.45 \mathrm{E}-33$

GO:0022626 Cytosolic ribosome

$18 \quad 3.96 \mathrm{E}-28$

GO:0015934 Large ribosomal subunit

$17 \quad 1.54 \mathrm{E}-25$

GO:0044445 Cytosolic part

$18 \quad 6.76 \mathrm{E}-22$

GO:0098798 Mitochondrial protein complex

$17 \quad 8.16 \mathrm{E}-20$

GO:0005743 Mitochondrial inner membrane

$19 \quad 2.01 \mathrm{E}-18$

GO:0022625 Cytosolic large ribosomal subunit

$11 \quad 1.14 \mathrm{E}-17$

\section{Molecular function}

GO:0003735 Structural constituent of ribosome $\quad 24 \quad 1.15 \mathrm{E}-33$

$\begin{array}{llll}\text { GO:0003954 NADH dehydrogenase activity } & 9 & 3.12 \mathrm{E}-14\end{array}$

GO:0008137 NADH dehydrogenase (ubiquinone) activity $\quad 9 \quad 3.12 \mathrm{E}-14$

$\begin{array}{llll}\text { GO:0050136 NADH dehydrogenase (quinone) activity } & 9 & 3.12 \mathrm{E}-14\end{array}$

GO:0016655 Oxidoreductase activity, acting on NAD $(\mathrm{P}) \mathrm{H}, \quad 9 \quad 3.26 \mathrm{E}-13$

quinone or similar compound as acceptor

GO:0016651 Oxidoreductase activity, acting on NAD(P)H $\quad 9 \quad 6.05 \mathrm{E}-11$

\begin{tabular}{llll} 
GO:0008009 Chemokine activity & 6 & $2.25 \mathrm{E}-08$ \\
\hline
\end{tabular}

\begin{tabular}{llll}
\hline KEGG pathway & & \\
\hline hsa03010 & Ribosome & 24 & $4.36 \mathrm{E}-29$ \\
hsa05171 & Coronavirus disease - COVID-19 & 21 & $2.35 \mathrm{E}-20$ \\
hsa00190 & Oxidative phosphorylation & 11 & $9.15 \mathrm{E}-10$ \\
\hline
\end{tabular}


Table 2 (on next page)

List of the hub genes involved in the 3 KEGG pathways

DCE: differentially co-expressed 


\begin{tabular}{|c|c|c|c|}
\hline $\mathbf{S} / \mathbf{N}$ & $\begin{array}{l}24 \text { differentially co- } \\
\text { expressed (DCE) } \\
\text { ribosome genes }\end{array}$ & $\begin{array}{l}21 \text { DCE coronavirus } \\
\text { disease - COVID-19 }\end{array}$ & $\begin{array}{l}11 \text { differentially co- } \\
\text { expressed oxidative } \\
\text { phosphorylation genes }\end{array}$ \\
\hline 1 & MRPS18A & RPL10L & UQCRQ \\
\hline 2 & RPL10L & RPL24 & NDUFB1 \\
\hline 3 & MRPL17 & RPS17 & NDUFB2 \\
\hline 4 & RPL24 & CXCL10 & NDUFB3 \\
\hline 5 & RPS17 & RPL30 & NDUFS6 \\
\hline 6 & RPL30 & RPS18 & NDUFA1 \\
\hline 7 & RPS18 & $\mathrm{C} 3$ & NDUFA3 \\
\hline 8 & RPS19 & RPS19 & NDUFA11 \\
\hline 9 & RPL29 & RPL29 & NDUFB9 \\
\hline 10 & RPL31 & RPL31 & NDUFA6 \\
\hline 11 & RPL36 & RPL36 & UQCR11 \\
\hline 12 & MRPL33 & RPS21 & \\
\hline 13 & RPS21 & FAU & \\
\hline 14 & MRPL21 & RPS24 & \\
\hline 15 & FAU & RPL14 & \\
\hline 16 & RPS24 & RPS25 & \\
\hline 17 & RPL14 & RPL22L1 & \\
\hline 18 & RPS25 & RPL11 & \\
\hline 19 & RPL22L1 & RPL35 & \\
\hline 20 & RPL35 & RPL12 & \\
\hline 21 & MRPL14 & RPS7 & \\
\hline 22 & RPL11 & & \\
\hline 23 & RPL12 & & \\
\hline 24 & RPS7 & & \\
\hline
\end{tabular}

1

2 


\section{Table 3 (on next page)}

List of the core hub genes involved in the 3 KEGG pathways. 


\begin{tabular}{|c|c|c|c|}
\hline KEGG pathway & $\begin{array}{c}\text { Number of } \\
\text { genes }\end{array}$ & List of genes & p. adjust \\
\hline hsa03010: Ribosome & 10 & $\begin{array}{l}\text { RPS24/RPS21/RPL31/RPL30/RPS17/FAU/RPS25/RP } \\
\text { L22L1/MRPL33/RPL11 }\end{array}$ & $3.12 \mathrm{E}-14$ \\
\hline $\begin{array}{l}\text { hsa05171: Coronavirus disease - } \\
\text { COVID-19 }\end{array}$ & 9 & $\begin{array}{l}\text { RPS24/RPS21/RPL31/RPL30/RPS17/FAU/RPS25/RP } \\
\text { L22L1/RPL11 }\end{array}$ & $6.89 \mathrm{E}-11$ \\
\hline $\begin{array}{l}\text { hsa04061: Viral protein interaction } \\
\text { with cytokine and cytokine receptor }\end{array}$ & 2 & CXCL9/CCL4 & 0.0552 \\
\hline
\end{tabular}

1

2 